\title{
HAUSDORFF DIMENSIONS OF SELF-SIMILAR AND SELF-AFFINE FRACTALS IN THE HEISENBERG GROUP
}

\section{ZOLTÁN M. BALOGH AND JEREMY T. TYSON}

\section{Introduction}

Analysis on the Heisenberg group is motivated by its appearance in several complex variables and quantum mechanics. In addition, as the simplest nonabelian example, the Heisenberg group serves as a testing ground for questions and conjectures on more general Carnot groups and sub-Riemannian spaces. Geometric measure theory and rectifiability play an important role in these settings in connection with sub-elliptic PDEs and control theory. For recent results in the subject we refer to $[\mathbf{3}, \mathbf{5}, \mathbf{1 2}, \mathbf{1 3}, \mathbf{1 5}, \mathbf{1 8}]$.

This paper is part of a larger program $[\mathbf{5}, \mathbf{4}]$ for studying properties of fractal sets in the sub-Riemannian metric setting of the Heisenberg group. The results presented here concern the Hausdorff dimensions of invariant sets associated to self-similar and self-affine iterated function systems.

Let us recall that the (first) Heisenberg group $\mathbb{H}=\mathbb{H}^{1}$ is the unique non-abelian Carnot group of rank 2 and dimension 3. Explicitly, $\mathbb{H}=\mathbb{R}^{3}$ with the group law

$$
(x, t) *\left(x^{\prime}, t^{\prime}\right)=\left(x+x^{\prime}, t+t^{\prime}+2\left\langle x, J x^{\prime}\right\rangle\right)
$$

where $J: \mathbb{R}^{2} \rightarrow \mathbb{R}^{2}$ denotes the map

$$
J\left(x_{1}, x_{2}\right)=\left(-x_{2}, x_{1}\right)
$$

and $\langle\cdot, \cdot\rangle$ is the standard inner product in $\mathbb{R}^{2}$.

The sub-Riemannian nature of $\mathbb{H}$ is reflected in the so-called horizontal distribution $H \mathbb{H}$, which is the distinguished subbundle of the full tangent bundle $T \mathbb{H}$ defined by

$$
H_{p} \mathbb{H}:=\operatorname{span}\left\{X_{p}, Y_{p}\right\} .
$$

Here $X$ and $Y$ denote the left-invariant vector fields in $\mathbb{H}$ whose values at a point $p=\left(x_{1}, x_{2}, t\right)$ are

$$
X_{p}=\partial_{x_{1}}+2 x_{2} \partial_{t}, \quad Y_{p}=\partial_{x_{2}}-2 x_{1} \partial_{t} .
$$

Equivalently, $H_{p} \mathbb{H}$ can be characterized as the kernel of the canonical contact form $d \tau=d t+2 x_{1} d x_{2}-2 x_{2} d x_{1}$ on $\mathbb{H}$ at the point $p$.

The Heisenberg group is equipped with a non-Euclidean metric structure via the so-called Heisenberg metric. This is the left-invariant metric on $\mathbb{H}$ defined

Received 16 March 2004; revised 9 September 2004.

2000 Mathematics Subject Classification 22E30, 28A78 (primary), 26A18, 28A78 (secondary).

Z. M. B. was supported by a grant from the Swiss NSF. J. T. T. was supported by NSF grant DMS 0228807. The research for this paper was done while J. T. T. was a visitor at the University of Berne during 2003. He wishes to thank the department for its hospitality. 
as follows:

$$
d_{H}(p, q)=\left|p^{-1} * q\right|_{H}, \quad \text { for } p, q \in \mathbb{H},
$$

where $*$ denotes the group law from $(1.1)$ and $|\cdot|_{H}$ denotes the Heisenberg norm given by

$$
|(x, t)|_{H}=\left(|x|^{4}+t^{2}\right)^{1 / 4} .
$$

Before passing to the main results of this paper, let us begin by describing an application which served as motivation for our studies.

The relationship between the Heisenberg and Euclidean geometry on $\mathbb{H}=\mathbb{R}^{3}$ is rather intricate. The Heisenberg Hausdorff dimension is always greater than or equal to its Euclidean counterpart; see, for example, (2.6). The inequality can be strict; indeed, the Hausdorff dimension of $\left(\mathbb{H}, d_{H}\right)$ is equal to 4 (in fact, balls in the metric $d_{H}$ have measure proportional to the fourth power of their radius). This implies, for instance, that the Heisenberg metric $d_{H}$ cannot be locally bi-Lipschitz equivalent with any Riemannian metric, in particular, with the Euclidean metric $d_{E}$.

A version of the following problem was posed by Gromov $[\mathbf{1 5}, 0.6 . \mathrm{C}]$ in the setting of general sub-Riemannian manifolds.

Problem 1.4. For fixed $\alpha \in[0,3]$, what are the possible values of $\beta=\operatorname{dim}_{H} S$ when $S$ ranges over all subsets of $\mathbb{H}$ with $\operatorname{dim}_{E} S=\alpha$ ?

Here and henceforth we denote by $\mathcal{H}_{H}^{s}$ and $\mathcal{H}_{E}^{s}$ the $s$-dimensional Hausdorff measures associated with the relevant metrics $d_{H}$ and $d_{E}$, respectively, and by $\operatorname{dim}_{H}$ and $\operatorname{dim}_{E}$ the corresponding Hausdorff dimensions.

Problem 1.4 is a fundamental question regarding the Hausdorff measures on $\mathbb{H}$ with respect to the Heisenberg metric. It asks which subsets of $\mathbb{H}$ are 'most nearly Euclidean' ( $\beta$ is smallest for fixed $\alpha$ ) and which are 'most nearly non-Euclidean' ( $\beta$ is largest for fixed $\alpha$ ). Recently, a nearly complete answer to Problem 1.4 was obtained by Balogh, Rickly and Serra-Cassano [5]. We formulate a slightly different version of the original statement in Theorems 1.1 and 1.2 of [5].

Theorem 1.5 (Balogh, Rickly and Serra-Cassano). Let $S \subset \mathbb{H}$ with

$$
\operatorname{dim}_{E} S=\alpha \in[0,3] \text { and } \operatorname{dim}_{H} S=\beta \in[0,4] .
$$

Then

$$
\max \{\alpha, 2 \alpha-2\}=: \beta_{-}(\alpha) \leqslant \beta \leqslant \beta_{+}(\alpha):=\min \{2 \alpha, \alpha+1\} .
$$

Moreover,

(i) for each $\alpha \in[0,3]$ there exists a set $S^{\alpha} \subset \mathbb{H}$ with $\mathcal{H}_{E}^{\alpha}\left(S^{\alpha}\right)<\infty$ and $\mathcal{H}_{H}^{\beta_{+}(\alpha)}\left(S^{\alpha}\right)>0$

(ii) for each $\alpha \in[0,2) \cup\{3\}$ there is a set $S_{\alpha} \subset \mathbb{H}$ with $\mathcal{H}_{E}^{\alpha}\left(S_{\alpha}\right)>0$ and $\mathcal{H}_{H}^{\beta_{-}(\alpha)}\left(S_{\alpha}\right)<\infty$, and

(ii $\left.^{\prime}\right)$ for each $\alpha \in[2,3)$ and each $\delta \in(0,1)$ there is a set $S_{\alpha, \delta} \subset \mathbb{H}$ with $\mathcal{H}_{E}^{\alpha-\delta}\left(S_{\alpha, \delta}\right)>0$ and $\mathcal{H}_{H}^{\beta_{-}(\alpha)}\left(S_{\alpha, \delta}\right)=\mathcal{H}_{H}^{2 \alpha-2}\left(S_{\alpha, \delta}\right)<\infty$.

See Figure 1.1 for the graphs of $\beta_{ \pm}(\alpha)$. Observe also the duality relation

$$
4-\beta_{+}(\alpha)=\beta_{-}(3-\alpha) \text {. }
$$




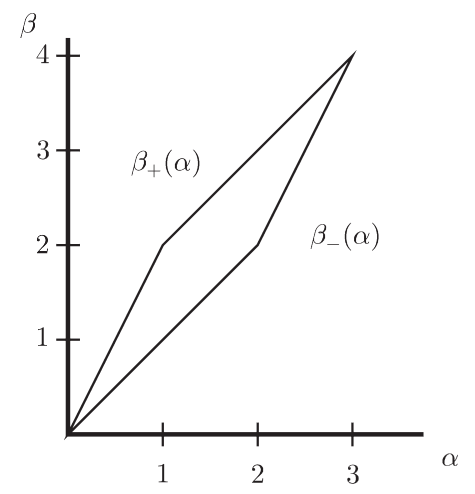

FiguRE 1.1. Hausdorff measure comparison functions $\beta_{ \pm}(\alpha)$ in the Heisenberg group.

The techniques in [5] did not suffice to obtain examples to show sharpness in the lower bound in (1.6) in the case $2 \leqslant \alpha<3$. In particular, [5] did not contain examples of sets $S$ with the property that

$$
\operatorname{dim}_{E} S=\operatorname{dim}_{H} S=2 .
$$

As a consequence of our main results (which we describe shortly) we are able to find such examples and complete the solution to Gromov's Problem 1.4. More precisely, we may record the following theorem.

Theorem 1.7. For each $\alpha \in[0,3]$ there exists $S_{\alpha} \subset \mathbb{H}$ with $\mathcal{H}_{E}^{\alpha}\left(S_{\alpha}\right)>0$ and $\mathcal{H}_{H}^{\beta_{-}(\alpha)}\left(S_{\alpha}\right)<\infty$, where $\beta_{-}(\alpha)=\max \{\alpha, 2 \alpha-2\}$.

The case $\alpha=\beta_{-}(\alpha)=2$ is of particular interest. The relevant example in this case is a self-similar set $Q_{H} \subset \mathbb{H}$ which we call the Heisenberg square. It is obtained as the invariant set for a certain self-similar iterated function system. Such systems are the main objects of study in this paper. We will describe this example in more detail later on in this introduction. Here let us give a few relevant facts which indicate how the proof of Theorem 1.7 goes. The 1-Lipschitz projection mapping $\pi: \mathbb{H} \rightarrow \mathbb{R}^{2}$ given by

$$
\pi(x, t)=x
$$

maps $Q_{H}$ onto the closed unit square $Q=[0,1]^{2}$. Thus $\mathcal{H}_{E}^{2}\left(Q_{H}\right) \geqslant \mathcal{H}_{E}^{2}(Q)=1>0$. On the other hand, the self-similar construction of $Q_{H}$ gives rise to natural coverings by families of self-similar copies of $Q_{H}$, and using these covers to estimate the Heisenberg Hausdorff measure yields $\mathcal{H}_{H}^{2}\left(Q_{H}\right)<\infty$.

The case $\alpha=2$ is the key to establishing Theorem 1.7 in full generality. The examples for $2<\alpha<3$ are constructed as certain 'product-type' sets using the Heisenberg square $Q_{H}$ together with vertical Cantor sets.

With this motivation in mind we turn to the principal objects of study in this paper, namely, invariant sets for iterated function systems in $\left(\mathbb{H}, d_{H}\right)$. Recall that an iterated function system (for short, an $\operatorname{IFS}$ ) on a complete metric space $(X, d)$ is a finite collection

$$
\mathcal{F}=\left\{f_{1}, \ldots, f_{M}\right\}
$$


of contraction maps of $(X, d)$, that is, Lipschitz maps with Lipschitz constant strictly less than 1 . The invariant set for $\mathcal{F}$ is the unique non-empty compact set in $X$ which is invariant under the action of the elements of $\mathcal{F}$. See (2.3).

In joint work with Regula Hofer-Isenegger [4], we studied regularity and connectivity questions for invariant sets of Heisenberg iterated function systems. The present work is devoted to the study of the dimensions of such invariant sets.

Throughout this paper, we restrict our attention to the case of affine iterated function systems (AIFS). That is, we assume that each IFS consists entirely of affine maps. Moreover, we are interested in affine contractions of $\mathbb{H}$ that arise as lifts of affine mappings of $\mathbb{R}^{2}$ as follows.

Let $f: \mathbb{R}^{2} \rightarrow \mathbb{R}^{2}$. A map $F: \mathbb{H} \rightarrow \mathbb{H}$ is called a (horizontal) lift of $f$ if

$$
\pi \circ F=f \circ \pi
$$

where $\pi$ is the map in (1.8). It is an important observation that each horizontal lift of an affine map of $\mathbb{R}^{2}$ which is Lipschitz with respect to $d_{H}$ is necessarily affine. Conversely, each affine map of $\mathbb{R}^{2}$ may be lifted to an affine Lipschitz map of $\mathbb{H}$. See Proposition 2.2 of [4] and $\S 2$ of this paper. Such lifts are not unique, but any two lifts of a given map of $\mathbb{R}^{2}$ differ only by the addition of a vertical constant.

Each AIFS $\mathcal{F}_{H}=\left\{F_{1}, \ldots, F_{M}\right\}$ on $\mathbb{H}$ therefore arises as a lift of an AIFS $\mathcal{F}=$ $\left\{f_{1}, \ldots, f_{M}\right\}$ on $\mathbb{R}^{2}$ and, conversely, each planar AIFS can be lifted to Heisenberg AIFSs. From the aforementioned ambiguity in the vertical constants, it follows that the space of all Heisenberg AIFSs which arise as lifts of a given planar AIFS $\mathcal{F}$ is naturally parameterized by an $M$-dimensional Euclidean space, where $M$ is the cardinality of $\mathcal{F}$.

We call the invariant sets for Heisenberg AIFSs (self-affine) horizontal fractals. This terminology comes from the fact that these objects are in some sense tangent to the horizontal distribution $H \mathbb{H}$. In this paper, we study the Hausdorff dimensions of horizontal fractals with respect to the metrics $d_{H}$ and $d_{E}$ on $\mathbb{H}=\mathbb{R}^{3}$.

To give a concrete example we describe in detail our basic example, the so-called Heisenberg square $Q_{H}$. By this name we denote the invariant set for any horizontal lift of the planar AIFS

$$
\mathcal{F}=\left\{f_{0}, f_{1}, f_{2}, f_{3}\right\},
$$

where $f_{j}(x)=\frac{1}{2}\left(x+e_{j}\right)$, for $j=0,1,2,3$. Here $e_{1}=(1,0)$ and $e_{2}=(0,1)$ are the standard basis vectors in $\mathbb{R}^{2}$, while $e_{0}=(0,0)$ and $e_{3}=e_{1}+e_{2}$. Figure 1.2 shows several versions of the Heisenberg square, corresponding to different lifts $\mathcal{F}_{H}$ of the IFS $\mathcal{F}$ from (1.9).
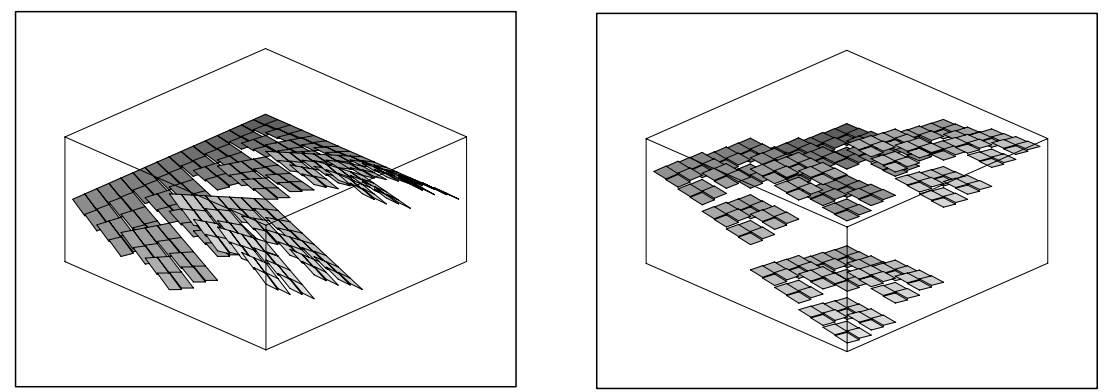

Figure 1.2. Heisenberg squares: horizontal lifts of $Q=[0,1]^{2}$. 
As indicated above, our first result gives the dimensions of Heisenberg squares.

TheOREm 1.10. Let $\mathcal{F}$ be the IFS in (1.9) and let $\mathcal{F}_{H}$ be any horizontal lift of $\mathcal{F}$. Denote by $Q=[0,1]^{2}$ and $Q_{H}$ the invariant sets for $\mathcal{F}$ and $\mathcal{F}_{H}$, respectively. Then

$$
\operatorname{dim}_{H} Q_{H}=\operatorname{dim}_{E} Q_{H}=\operatorname{dim}_{E} Q=2 .
$$

In fact we have

$$
0<1=\mathcal{H}_{E}^{2}(Q) \leqslant \mathcal{H}_{E}^{2}\left(Q_{H}\right) \quad \text { and } \quad \mathcal{H}_{H}^{2}\left(Q_{H}\right)<\infty .
$$

Observe that (1.11) follows from (1.12) and (2.6).

The Heisenberg squares have been considered previously. Strichartz [24] used $Q_{H}$ (and versions in more general Carnot groups) to construct 'dyadic-type' Carnot tilings. See also [25]. The equality $\operatorname{dim}_{H} Q_{H}=2$ in Theorem 1.10 can be found in [24]. However, Strichartz obtained $Q_{H}$ in a different way as the graph of an $L^{\infty}$-function and not as a horizontal lift. Due to our different approach we obtain a more complete statement and a much simpler proof of Theorem 1.10. Indeed, we will shortly describe a significantly more general result from which Theorem 1.10 arises as an easy corollary.

Let us mention that the Heisenberg square $Q_{H}$ is also interesting for another reason. In [4], we prove the following result: there exists a horizontal lift $\mathcal{F}_{H}$ of the IFS $\mathcal{F}$ from (1.9), so that each selection $\beta: Q \rightarrow \mathbb{H}, \beta(x)=(x, g(x))$, of the set-valued map $\alpha(x)=\pi^{-1}(x) \cap Q_{H}$, is a function of bounded variation. Combining this result and Theorem 1.10, we see that there exists a surface $S=g(\operatorname{int} Q)$ in $\mathbb{H}$ with

$$
0<\mathcal{H}_{H}^{2}(S)<\infty
$$

and $g$ a function of bounded variation. By way of contrast, Ambrosio and Kirchheim [1, Theorem 7.2] have shown that there are no Lipschitz horizontal surfaces in $\mathbb{H}$, that is, surfaces $S=g(\Omega)$, with $\Omega \subset \mathbb{R}^{2}$, which satisfy (1.13) with $\beta=(\mathrm{id}, g)$ a Lipschitz map from $\Omega$ to $\left(\mathbb{H}, d_{H}\right)$.

As mentioned above, Theorem 1.10 is a special case of more general results concerning the dimensions of self-similar and self-affine horizontal fractals. The results in question are Heisenberg analogs of theorems of Falconer [10] and Solomyak $[\mathbf{2 3}]$ on the dimensions of generic invariant sets. To set the stage we recall in brief some results from $[\mathbf{1 0}]$ and $[\mathbf{2 3}]$. A more detailed description can be found in $\S 5$.

To a finite collection $\mathcal{A}$ of contractive linear maps of $\mathbb{R}^{n}$, Falconer [10] associates a critical exponent $s_{E}(\mathcal{A})$. In the case when each element of $\mathcal{A}$ is in the conformal group $C O(n)=\mathbb{R}_{+} \times O(n)$, the critical exponent of $\mathcal{A}$ is equal to the similarity dimension of $\mathcal{A}$, that is, the unique value $s$ satisfying the equation

$$
\sum_{A \in \mathcal{A}}\|A\|^{s}=1
$$

where $\|\cdot\|$ denotes the operator norm. (It is not required that the elements of $\mathcal{A}$ be distinct.)

In the case of self-similar AIFSs satisfying the open set condition (cf. $\S 2$ ) we have the following remarkable equality of dimensions, which holds for every horizontal lift. 
THEOREM 1.15. Let $\mathcal{F}$ be a self-similar planar AIFS which satisfies the open set condition and let $\mathcal{F}_{H}$ be a horizontal lift of $\mathcal{F}$. Then

$$
\operatorname{dim}_{E} K=\operatorname{dim}_{E} K_{H}=\operatorname{dim}_{H} K_{H}=s,
$$

where $s$ denotes the similarity dimension for the associated family of conformal matrices. Moreover,

$$
0<\mathcal{H}_{E}^{s}(K) \leqslant \mathcal{H}_{E}^{s}\left(K_{H}\right) \text { and } \mathcal{H}_{H}^{s}\left(K_{H}\right)<\infty .
$$

Since the IFS $\mathcal{F}$ from (1.9) satisfies the open set condition, Theorem 1.10 follows from Theorem 1.15.

The major question which we address in this paper is what happens in the absence of the open set condition in the more general setting of affine maps. The definition of the critical exponent $s_{E}(\mathcal{A})$ from $[\mathbf{1 0}]$ is more complicated and will be recalled in $\S 5$. By results of Falconer and Solomyak in the Euclidean case one still has a dimension formula which holds in a generic sense. To recall this statement fix a collection $\mathcal{A}=\left\{A_{1}, \ldots, A_{M}\right\}$ as above. For each $b=\left(b_{1}, \ldots, b_{M}\right)$ in $\mathbb{R}^{n M}$, define an AIFS $\mathcal{F}(b)=\left\{f_{1}, \ldots, f_{M}\right\}$ on $\mathbb{R}^{n}$, where $f_{i}(x)=A_{i} x+b_{i}$, for $i=1, \ldots, M$. Let $K(b)$ be the invariant set for $\mathcal{F}(b)$.

Theorem 1.16 (Falconer, Solomyak). Let $\mathcal{A}$ and $K(b)$ be as above. Then

(i) $\operatorname{dim}_{E} K(b) \leqslant s_{E}(\mathcal{A})$ for all $b \in \mathbb{R}^{n M}$; and

(ii) if $\left\|A_{i}\right\|<\frac{1}{2}$ for each $i$, then $\operatorname{dim}_{E} K(b)=\min \left\{n, s_{E}(\mathcal{A})\right\}$ for a.e. $b \in \mathbb{R}^{n M}$.

Falconer proved Theorem 1.16 first with $\frac{1}{2}$ replaced by $\frac{1}{3}[\mathbf{1 0}$, Proposition 5.1 and Theorem 5.3]. Solomyak [23, Proposition 3.1] observed that the hypotheses could be weakened as indicated. The constant $\frac{1}{2}$ is sharp for generic statements of this type, as was observed by Edgar in [8]. See also the proof of Proposition 3.1 in [23].

Each lift of an affine map $f(x)=A x+b$ of $\mathbb{R}^{2}$ to the Heisenberg group is an affine map $F(x, t)=\widetilde{A}_{b}(x, t)+\widetilde{b}$, where $\widetilde{A}_{b}$ is a certain block-lower triangular matrix defined in terms of $A$ and $b$ and $\widetilde{b}=(b, \tau)$, with $\tau$ an arbitrary real parameter. See $(2.2)$. For a given $b \in \mathbb{R}^{2 M}$ and an AIFS $\mathcal{F}(b)$ on $\mathbb{R}^{2}$, denote by $\mathcal{F}_{H}(b, \tau)$ the lifted AIFS corresponding to a specific choice of $\tau \in \mathbb{R}^{M}$, and denote by $K_{H}(b, \tau)$ its invariant set. Also, denote by $\widetilde{s}_{E}(b ; \mathcal{A})$ the critical exponent for the family $\left\{\widetilde{A}_{1, b_{1}}, \ldots, \widetilde{A}_{M, b_{M}}\right\}$.

From Theorem 1.16 we immediately deduce that

$$
\operatorname{dim}_{E} K_{H}(b, \tau) \leqslant \widetilde{s}_{E}(b ; \mathcal{A})
$$

for all $b$ and $\tau$. However, the upper bound in (1.17) is not the correct value for $\operatorname{dim}_{E} K_{H}(b, \tau)$. In fact, we will prove the following result.

ThEOREM 1.18. Let $\mathcal{F}(b)$, with $b \in \mathbb{R}^{2 M}$, be a planar AIFS and let $\mathcal{F}_{H}(b, \tau)$, with $\tau \in \mathbb{R}^{M}$, be any horizontal lift. Then

(i) $\operatorname{dim}_{E} K_{H}(b, \tau) \leqslant \widetilde{s}_{E}(\mathcal{A}):=\widetilde{s}_{E}(0 ; \mathcal{A})$ for all $b \in \mathbb{R}^{2 M}$ and $\tau \in \mathbb{R}^{M}$; and

(ii) if $\left\|A_{i}\right\|<\frac{1}{2}$ for all $i$, then $\operatorname{dim}_{E} K_{H}(b, \tau)=\min \left\{3, \widetilde{s}_{E}(\mathcal{A})\right\}$ for a.e. $b \in \mathbb{R}^{2 M}$ and $\tau \in \mathbb{R}^{M}$.

Observe that there is no contradiction between the almost sure results of Theorems 1.18(ii) and 1.16(ii) since the matrices $\widetilde{A}_{i, b_{i}}$ depend on the auxiliary parameter 
$b \in \mathbb{R}^{2 M}$. Thus it cannot be guaranteed that the almost sure conclusion in Theorem 1.16 is applicable for any particular choice of the lift $K_{H}(b, \tau)$ in Theorem 1.18(ii).

To study the Heisenberg dimensions of $K_{H}(b, \tau)$ we introduce a Heisenberg critical exponent $\widetilde{s}_{H}(\mathcal{A})$ associated with a family $\mathcal{A}$ of contractive linear maps of $\mathbb{R}^{2}$. This quantity differs substantially from its Euclidean counterpart and represents a major conceptual novelty of this paper. We then have the following result.

Theorem 1.19. Let $\mathcal{F}(b)$, with $b \in \mathbb{R}^{2 M}$, be a planar AIFS and let $\mathcal{F}_{H}(b, \tau)$, with $\tau \in \mathbb{R}^{M}$, be any horizontal lift. Then

(i) $\operatorname{dim}_{H} K_{H}(b, \tau) \leqslant \widetilde{s}_{H}(\mathcal{A})$ for all $b \in \mathbb{R}^{2 M}$ and $\tau \in \mathbb{R}^{M}$; and

(ii) if $\left\|A_{i}\right\|<\frac{1}{2}$ for each $i$, then $\operatorname{dim}_{H} K_{H}(b, \tau)=\min \left\{4, \widetilde{s}_{H}(\mathcal{A})\right\}$ for almost every $b \in \mathbb{R}^{2 M}$ and $\tau \in \mathbb{R}^{M}$.

From the definitions of $s_{E}, \widetilde{s}_{E}$ and $\widetilde{s}_{H}$ it is straightforward to verify that

$$
\min \left\{2, s_{E}(\mathcal{A})\right\} \leqslant \min \left\{3, \widetilde{s}_{E}(\mathcal{A})\right\} \leqslant \min \left\{4, \widetilde{s}_{H}(\mathcal{A})\right\} .
$$

Furthermore, if $0 \leqslant s_{E}(\mathcal{A}) \leqslant 1$ then $s_{E}(\mathcal{A})=\widetilde{s}_{E}(\mathcal{A})=\widetilde{s}_{H}(\mathcal{A})$ and if $1 \leqslant s_{E}(\mathcal{A}) \leqslant 2$ then $s_{E}(\mathcal{A})=\widetilde{s}_{E}(\mathcal{A})$.

In the self-similar case, the critical exponents $s_{E}(\mathcal{A}), \widetilde{s}_{E}(\mathcal{A})$ and $\widetilde{s}_{H}(\mathcal{A})$ all agree and are equal to the similarity dimension of $\mathcal{A}$. Denoting this common value by $s$, we have

$$
\begin{gathered}
\operatorname{dim}_{E}(K(b))=\min \{2, s\}, \\
\operatorname{dim}_{E}\left(K_{H}(b, \tau)\right)=\min \{3, s\},
\end{gathered}
$$

and

$$
\operatorname{dim}_{H}\left(K_{H}(b, \tau)\right)=\min \{4, s\}
$$

for almost every $b$ and $\tau$. In particular, if $s \leqslant 2$ then

$$
\operatorname{dim}_{E} K(b)=\operatorname{dim}_{E} K_{H}(b, \tau)=\operatorname{dim}_{H} K_{H}(b, \tau)=s
$$

for a.e. $b$ and $\tau$.

Note added in October 2004

Theorems 1.5 and 1.7 state that the map $S \mapsto\left(\operatorname{dim}_{E} S, \operatorname{dim}_{H} S\right)$ from subsets of $\mathbb{H}$ into $\mathbb{R}^{2}$ has range contained in the (closed) parallelogram $\Pi$ shown in Figure 1.1, and that the boundary of this parallelogram is contained in this range. In fact, it is easy to see that the range of this map coincides with the parallelogram, that is, for every $(\alpha, \beta) \in \Pi$ there exists a set $S \subset \mathbb{H}$ with $\operatorname{dim}_{E} S=\alpha$ and $\operatorname{dim}_{H} S=\beta$. Indeed, the monotonicity of the functions $\beta_{+}(\alpha)$ and $\beta_{-}(\alpha)$ ensures that the set

$$
S=S_{\alpha} \cup S^{\beta_{+}^{-1}(\beta)}
$$

has the desired property, where $S^{\alpha}$ and $S_{\alpha}$ are the sets constructed in Theorems 1.5 and 1.7 , respectively.

\section{Overview}

The structure of this paper is as follows. In $\S 2$ we collect some definitions and recall background material. We also fix notation which will be in force for the rest of the paper. 
Section 3 is devoted to the self-similar case. We prove Theorems 1.18 and 1.19 in this special setting first, in preparation for the general case. We also discuss the open set condition for horizontal lifts, and give the proof of Theorem 1.15.

In $\S 4$ we discuss Gromov's question on the relationship between $\operatorname{dim}_{E}$ and $\operatorname{dim}_{H}$.

The various critical exponents for a general affine family and its horizontal lifts are defined and discussed in $\S 5$. Section 6 is devoted to the proofs of Theorems 1.18 and 1.19 in complete generality.

In an appendix, we sketch the proof of an interesting fact from linear algebra which arises in connection with inequalities between the various critical exponents associated with a family of contractive linear maps.

\section{Definitions, notation and preliminary results}

\subsection{Affine maps on $\mathbb{H}$}

We start by recalling the following relation between affine maps of $\mathbb{H}$ and Lipschitz horizontal lifts of affine maps of $\mathbb{R}^{2}$. See Proposition 2.2 and Theorem 1.6 of $[4]$. Let $F: \mathbb{R}^{3} \rightarrow \mathbb{R}^{3}$ be an affine map of the form

$$
F(x, t)=(A x+t a+b,\langle d, x\rangle+c t+\tau),
$$

where $A$ is a real $2 \times 2$ matrix, $a, b, d \in \mathbb{R}^{2}$ and $c, \tau \in \mathbb{R}$. Then $F$ is Lipschitz with respect to the metric $d_{H}$ if and only if the relations

$$
a=0, \quad d=-2 A^{T} J b, \quad c=\operatorname{det} A
$$

hold. Thus every Lipschitz affine map $F: \mathbb{H} \rightarrow \mathbb{H}$ may be written as

$$
F(x, t)=\widetilde{A}_{b}\left(\begin{array}{l}
x \\
t
\end{array}\right)+\widetilde{b},
$$

where

$$
\widetilde{A}_{b}=\left(\begin{array}{cc}
A & 0 \\
-2(J b)^{T} A & \operatorname{det} A
\end{array}\right), \quad \widetilde{b}=\left(\begin{array}{c}
b \\
\tau
\end{array}\right),
$$

and $\tau$ is a real constant. In particular, $F$ is a horizontal lift of the affine map $f(x)=A x+b$. Moreover, any Lipschitz horizontal lift of $f$ is necessarily an affine map of the form (2.1). The Lipschitz constant of $F$ as a map of $\left(\mathbb{H}, d_{H}\right)$ is equal to the Lipschitz constant of $f$ as a map of $\left(\mathbb{R}^{2}, d_{E}\right)$. Furthermore, $F$ is a similarity with respect to $d_{H}$ if and only if the above relations hold and $A \in C O(2)$ is a conformal matrix. In this case the Lipschitz constant agrees with the operator norm of the linear part of $f$.

For example, choose $A=r I$, with $r>0$ (where $I$ denotes the $2 \times 2$ identity matrix), and $b=0$. The lift of $f(x)=r x$ corresponding to $\tau=0$ is the Heisenberg dilation $F(x, t)=\left(r x, r^{2} t\right)$. Similarly, choose $A=I$ and $b \in \mathbb{R}^{2}$ arbitrarily. Then the lift of $f(x)=x+b$ corresponding to $\tau \in \mathbb{R}$ is the left translation by $(b, \tau)$ :

$$
F(x, t)=(b, \tau) *(x, t)=(x+b, t+\tau-2\langle J b, x\rangle) .
$$

\subsection{Affine iterated function systems}

Let $X$ be either $\mathbb{R}^{n}$, with $n=2,3$, or $\mathbb{H}$. Recall that an affine iterated function system $(A I F S)$ is a finite collection $\mathcal{F}$ of contracting affine maps of $X$. The 
invariant set for $\mathcal{F}$ is the unique non-empty compact set $K \subset X$ which is fully invariant under the action of $\mathcal{F}$ :

$$
K=\bigcup_{f \in \mathcal{F}} f(K) .
$$

The existence of invariant sets for iterated function systems follows from the completeness of the space of compact subsets of $X$ with the Hausdorff metric. See, for example, [19, 4.13] or [17, Theorem 1.1.4].

It follows from the previous paragraph that, to each planar AIFS

$$
\mathcal{F}(b)=\left\{f_{1}, \ldots, f_{M}\right\},
$$

where $b=\left(b_{1}, \ldots, b_{M}\right) \in \mathbb{R}^{2 M}$ and $f_{i}(x)=A_{i} x+b_{i}$, there correspond Heisenberg AIFSs given by

$$
\mathcal{F}_{H}(b, \tau)=\left\{F_{1}, \ldots, F_{M}\right\}
$$

where $\tau=\left(\tau_{1}, \ldots, \tau_{M}\right) \in \mathbb{R}^{M}$,

$$
F_{i}(x, t)=\widetilde{A}_{i, b_{i}}\left(\begin{array}{c}
x \\
t
\end{array}\right)+\widetilde{b}_{i},
$$

and $\widetilde{A}_{i, b_{i}}$ and $\widetilde{b}_{i}$ are given in analogy with (2.2). We call such a system $\mathcal{F}_{H}(b, \tau)$ a horizontal lift of $\mathcal{F}(b)$. Throughout this paper we denote by $K(b)$ and $K_{H}(b, \tau)$ the invariant sets for $\mathcal{F}(b)$ and $\mathcal{F}_{H}(b, \tau)$, respectively. We also call $K_{H}(b, \tau)$ a horizontal lift of $K(b)$. The space of all horizontal lifts $\mathcal{F}_{H}(b, \tau)$ of a fixed AIFS $\mathcal{F}(b)$ of cardinality $M$ depends on the $M$ real parameters $\tau_{1}, \ldots, \tau_{M}$.

We call an AIFS $\mathcal{F}(b)$ or $\mathcal{F}(b, \tau)$ self-similar if the matrices $A_{i}$ or $\widetilde{A}_{i, b_{i}}$ are conformal. Recall that the similarity dimension of $\mathcal{A}=\left\{A_{1}, \ldots, A_{M}\right\}$ is the unique positive solution $s$ to the equation (1.14). From remarks made in the previous paragraph, it follows that the Heisenberg similarity dimension of the family $\left\{\widetilde{A}_{1, b_{1}}, \ldots, \widetilde{A}_{M, b_{M}}\right\}$ is equal to the same value $s$, regardless of the choice of $b_{1}, \ldots, b_{M}$.

\subsection{Symbolic dynamics}

The dynamical attributes of an iterated function system are encoded via its representation as a quotient of sequence space. Let $\mathfrak{A}$ be an alphabet consisting of the letters $1, \ldots, M$. Let $W_{m}=\mathfrak{A}^{m}$, for $m \geqslant 1$, denote the space of words of length $m$, and let $\Sigma=\mathfrak{A}^{\mathbb{N}}$ denote the space of words of infinite length, with letters drawn from $\mathfrak{A}$ in both cases. We denote elements of these spaces by concatenation of letters, that is, $w=w_{1} w_{2} \ldots w_{m} \in W_{m}$ or $w=w_{1} w_{2} \ldots \in \Sigma$, where $w_{j} \in \mathfrak{A}$ for each $j$. Let $W=\bigcup_{m \geqslant 1} W_{m}$ be the collection of all words of finite length. For $w \in W$ we write $\Sigma_{w}$ for the set of words in $\Sigma$ which begin with $w ; \Sigma_{w}$ is called the cylinder set with label $w$.

Assume now that $\mathcal{F}=\left\{f_{i}\right\}_{i \in \mathfrak{A}}$ is an IFS in a complete metric space $(X, d)$ with invariant set $K$. For each finite word $w=w_{1} \ldots w_{m}$ let $f_{w}=f_{w_{1}} \circ \ldots \circ f_{w_{m}}$ and $K_{w}=f_{w}(K)$. Then $K=\bigcup_{w \in W_{m}} K_{w}$ for each $m$ and $\max _{w \in W_{m}} \operatorname{diam} K_{w} \rightarrow 0$ as $m \rightarrow \infty$. We also define $K_{w}$ for infinite words $w=w_{1} w_{2} \ldots$ by setting $K_{w}=\bigcap_{m} K_{w_{1} \ldots w_{m}}$. In this case $K_{w}$ consists of a single point in $K$.

We consider on $\Sigma$ the product topology induced by the discrete topology on $\mathfrak{A}$ and we define a map $p=p_{\mathcal{F}}: \Sigma \rightarrow K$ by setting $p(w)$ equal to the unique point in $K_{w}$. Then $p$ is a continuous surjection between compact sets [17, Theorem 1.2.3]. 
Observe that

$$
p(w)=\lim _{m \rightarrow \infty} f_{w_{1} \ldots w_{m}}\left(x_{0}\right), \quad \text { with } w=w_{1} w_{2} \ldots \in \Sigma,
$$

where $x_{0}$ is an arbitrarily chosen point in $X$.

\subsection{Hausdorff measure and dimension}

Let $X=(X, d)$ be a metric space. For $\alpha \geqslant 0$ we denote by $\mathcal{H}_{d}^{\alpha}$ the $\alpha$-dimensional Hausdorff measure on $X$, defined as

$$
\mathcal{H}_{d}^{\alpha}(A):=\liminf _{\delta \searrow 0} \sum_{n} \operatorname{diam}\left(A_{n}\right)^{\alpha},
$$

where the infimum is taken over all countable covers of $A$ by sets $A_{1}, A_{2}, \ldots$ satisfying $\operatorname{diam} A_{n}<\delta$. Then the Hausdorff dimension of $A \subset X$ is

$$
\operatorname{dim}_{d}(A)=\inf \left\{\alpha: \mathcal{H}_{d}^{\alpha}(A)=0\right\}=\sup \left\{\alpha: \mathcal{H}_{d}^{\alpha}(A)=\infty\right\} .
$$

We will use these concepts only in the cases $(X, d)=\left(\mathbb{R}^{2}, d_{E}\right),(X, d)=\left(\mathbb{R}^{3}, d_{E}\right)$, and $(X, d)=\left(\mathbb{H}, d_{H}\right)$. We write $\mathcal{H}_{E}^{\alpha}$ and $\operatorname{dim}_{E}$ for the Hausdorff measures and dimension in $\mathbb{R}^{2}$ and $\mathbb{R}^{3}$ and $\mathcal{H}_{H}^{\alpha}$ and $\operatorname{dim}_{H}$ for the corresponding objects in $\mathbb{H}$.

Since $d_{E}$ is locally bounded by $d_{H}$ on $\mathbb{H}=\mathbb{R}^{3}[\mathbf{5}$, Lemma 2.1], we have the absolute continuity relation $\mathcal{H}_{E}^{\alpha} \ll \mathcal{H}_{H}^{\alpha}$ for the $\alpha$-Hausdorff measures on $\mathbb{H}$ for any $\alpha \geqslant 0$ [5, Proposition 3.2(i)]. Thus

$$
\operatorname{dim}_{E} A \leqslant \operatorname{dim}_{H} A
$$

for any set $A \subset \mathbb{H}$.

\subsection{The open set condition}

An iterated function system $\mathcal{F}$ on a complete metric space $X$ is said to satisfy the open set condition if there exists a bounded open set $O \subset X$ so that $f(O) \subset O$ for all $f \in \mathcal{F}$ and $f(O) \cap g(O)=\emptyset$ for all $f, g \in \mathcal{F}$ with $f \neq g$.

The relevance of this condition for the computation of Hausdorff dimensions derives from the following result, which was proved by Moran [20] in 1946 and rediscovered by Hutchinson [16] in the 1980s. The class of self-similar AIFSs was defined in $\S 2.2$.

Proposition 2.7. Let $\mathcal{F}$ be a self-similar AIFS in $\mathbb{R}^{n}$ which satisfies the open set condition. Let $K$ be the invariant set of $\mathcal{F}$. Let $\mathcal{A}$ denote the collection of conformal matrices which arise as the linear parts of elements of $\mathcal{F}$ (counted with multiplicity).

Then the Hausdorff dimension of $K$ is equal to the similarity dimension $s$ of $\mathcal{A}$. Moreover,

$$
0<\mathcal{H}_{E}^{s}(K)<\infty
$$

Schief [22], building on ideas of Bandt and Graf [7], proved the following (somewhat surprising) converse to Proposition 2.7.

Proposition 2.8. Let $\mathcal{F}$ be a self-similar AIFS in $\mathbb{R}^{n}$ whose invariant set $K$ satisfies $\mathcal{H}_{E}^{s}(K)>0$, where $\mathcal{A}$ is defined as in Proposition 2.7. Then $\mathcal{F}$ satisfies the open set condition. 


\section{Self-similar horizontal fractals}

In this section, we discuss relations between the Euclidean dimension of a planar self-similar invariant set and the Heisenberg dimensions of its horizontal lifts. In particular, we prove Theorem 1.15 on the equality of dimensions in the presence of the open set condition. The principal theorem of this section (Theorem 3.9) states that the Heisenberg and Euclidean dimensions agree generically. It is a special case of Theorems 1.18 and 1.19 .

Throughout this section, we assume that $\mathcal{F}$ is a self-similar planar AIFS with invariant set $K$, and that $\mathcal{F}_{H}$ is a horizontal lift of $\mathcal{F}$ with invariant set $K_{H}$.

Since $\pi$ is a 1-Lipschitz map from $\left(\mathbb{R}^{3}, d_{E}\right)$ to $\left(\mathbb{R}^{2}, d_{E}\right)$ and $\pi\left(K_{H}\right)=K$, we have the following a priori inequality:

$$
\operatorname{dim}_{E} K \leqslant \operatorname{dim}_{E} K_{H} \leqslant \operatorname{dim}_{H} K_{H}
$$

Observe that the second inequality follows from (2.6).

The following example shows that we need not always have equality throughout (3.1). In this example it is the first equality which is strict.

EXAmPLE 3.2. Fix $r \in\left(\frac{1}{2}, 1 / \sqrt{2}\right)$ and let $f_{1}(x)=r x$ and $f_{2}(x)=e_{1}+r\left(x-e_{1}\right)$, where $e_{1}=(1,0)$. The invariant set for $\mathcal{F}=\left\{f_{1}, f_{2}\right\}$ is $[0,1]$. The formula in $(2.4)$ gives the horizontal lifts $F_{i}$, for $i=1,2$, as

$$
\begin{aligned}
& F_{1}(x, t)=\left(r x, r^{2} t+\tau_{1}\right), \\
& F_{2}(x, t)=\left(e_{1}+r\left(x-e_{1}\right), r^{2} t-2 r(1-r) x_{2}+\tau_{2}\right),
\end{aligned}
$$

where $x=\left(x_{1}, x_{2}\right)$ and $\tau_{1}, \tau_{2} \in \mathbb{R}$. Choose $\tau_{1}=0$ and

$$
\tau_{2}>\frac{2 r(1-r)}{1-2 r^{2}}
$$

It is straightforward to show that the open set $U=B(0,1) \times\left(0,2 \tau_{2}\right)$ satisfies the open set condition for $\mathcal{F}_{H}=\left\{F_{1}, F_{2}\right\}$. The maps $F_{1}$ and $F_{2}$ are similarities of $\mathbb{H}$ with contraction ratio $r$. By Proposition 3.3 below,

$$
\operatorname{dim}_{H} K_{H}=\frac{\log 2}{\log 1 / r}>1=\operatorname{dim}_{E} K .
$$

In fact, the Euclidean dimension of $K_{H}$ is also equal to $\log 2 / \log 1 / r$. The proof of this latter fact requires Falconer's theory of dimensions of self-affine fractals which will be recalled in $\S 5$.

In the above example we made use of the following proposition, which extends the Moran-Hutchinson result to the Heisenberg setting.

Proposition 3.3. Let $\mathcal{F}_{H}$ be a self-similar Heisenberg AIFS which satisfies the open set condition. Assume that $\mathcal{F}_{H}$ is a lift of $\mathcal{F}$, and define $\mathcal{A}$ as in Proposition 2.7. Then the Heisenberg dimension of $K_{H}$ is equal to the similarity dimension of $\mathcal{A}$.

Kigami [17, Proposition 1.5.8] gave a new proof of the theorem of Moran and Hutchinson. His proof extends to the Heisenberg setting, as we now demonstrate.

Kigami's proof uses the following more general result, which is Theorem 1.5.7 of $[\mathbf{1 7}]$. 
Theorem 3.4. Let $\mathcal{F}=\left\{f_{1}, \ldots, f_{M}\right\}$ be an iterated function system in a complete metric space $X$. Let $K$ be the invariant set of $\mathcal{F}$. Assume that there exist $r_{1}, \ldots, r_{M} \in(0,1)$ and positive constants $C_{1}, C_{2}, L$ and $r_{0}$ so that the following two conditions hold:

(i) $\operatorname{diam} f_{w}(K) \leqslant C_{1} r_{w}$ for each $w \in W$, and

(ii) for any $p \in K$ and any $0<r \leqslant r_{0}$, the number of words $w=w_{1} \ldots w_{m} \in W$ satisfying the conditions

$$
r_{w_{1}} \ldots r_{w_{m-1}}>r \geqslant r_{w}:=r_{w_{1}} \ldots r_{w_{m}}
$$

and

$$
\operatorname{dist}\left(p, f_{w}(K)\right) \leqslant C_{2} r
$$

is at most $L$, independent of $p$ and $r$.

Then the Hausdorff dimension of $K$ is given by the unique positive solution $s$ to the equation

$$
\sum_{i=1}^{M} r_{i}^{s}=1
$$

Moreover, $0<\mathcal{H}^{s}(K)<\infty$.

Proof of Proposition 3.3. We verify the assumptions of Theorem 3.4 with $r_{i}$ equal to the Lipschitz constant of $F_{i} \in \mathcal{F}_{H}$. Let $r_{\min }$ be the minimum of the $r_{i}$.

Let $U$ be a bounded open set in $\mathbb{H}$ which satisfies the open set condition for $\mathcal{F}_{H}$. Without loss of generality we may assume that $\operatorname{diam}_{H} \bar{U}=1$; since $K_{H} \subset \bar{U}$ by Exercise 1.2 of [17], we conclude that $\operatorname{diam}_{H} K_{H} \leqslant 1$.

By the choice of $r_{i}, \operatorname{diam}_{H} F_{w}\left(K_{H}\right) \leqslant r_{w}$ for all words $w$. This establishes Theorem 3.4(i) with $C_{1}=1$.

Next fix $p=(x, t) \in K_{H}$ and $0<r \leqslant 1$, and consider a word $w$ satisfying (3.5) and (3.6) with $C_{2}=1$. Then $F_{w}(U) \subset B_{H}(p, 2 r)$, where $B_{H}(p, r)$ denotes the ball in the Heisenberg metric about $p$ of radius $r$. Since the sets $F_{w}(U)$ are pairwise disjoint for such words $w$,

$$
\sum_{w}\left|F_{w}(U)\right| \leqslant\left|B_{H}(p, 2 r)\right|=16 r^{4}\left|B_{H}(0,1)\right|,
$$

where the sum is taken over all words $w$ satisfying (3.5) and (3.6). Here $|U|$ denotes the three-dimensional Lebesgue measure of a set $U \subset \mathbb{H}$. From (3.5) we see that $\left|F_{w}(U)\right| \geqslant r_{\min }^{4} r^{4}|U|$ and so the number of words $w$ is bounded by

$$
L:=\frac{16\left|B_{H}(0,1)\right|}{r_{\min }^{4}|U|} .
$$

Since the open set condition passes to horizontal lifts (see Proposition 3.14 of [4]), we may record the following corollary to Proposition 3.3, stated earlier as Theorem 1.15 .

Corollary 3.8. Let $\mathcal{F}$ be a self-similar planar AIFS which satisfies the open set condition and let $\mathcal{F}_{H}$ be a horizontal lift of $\mathcal{F}$. Then

$$
\operatorname{dim}_{E} K=\operatorname{dim}_{E} K_{H}=\operatorname{dim}_{H} K_{H}=s,
$$

where $s$ denotes the similarity dimension for the associated family of conformal 
matrices. Moreover,

$$
0<\mathcal{H}_{E}^{s}(K) \leqslant \mathcal{H}_{E}^{s}\left(K_{H}\right) \text { and } \quad \mathcal{H}_{H}^{s}\left(K_{H}\right)<\infty
$$

Generic equality of dimensions for self-similar fractals

In this subsection, we show that equality holds throughout (3.1) in a generic sense even in the absence of the open set condition.

Consider a family $\mathcal{A}=\left\{A_{1}, \ldots, A_{M}\right\}$ of $2 \times 2$ conformal matrices. For each $b=\left(b_{1}, \ldots, b_{M}\right) \in \mathbb{R}^{2 M}$, consider the AIFS $\mathcal{F}(b)=\left\{f_{1}, \ldots, f_{M}\right\}$, where $f_{i}(x)=$ $A_{i} x+b_{i}$, for $i=1, \ldots, M$. We view the matrices $A_{1}, \ldots, A_{M}$ as fixed and $b_{i}, \ldots, b_{M}$ as varying.

The following theorem gives an upper bound for the Hausdorff dimensions of self-similar lifts. In conjunction with Theorem 1.16, it implies the generic equality of Heisenberg and Euclidean dimensions.

TheOREM 3.9. Let $\mathcal{F}(b)$ be a self-similar planar AIFS as above, and let $\mathcal{F}_{H}(b, \tau)$, with $\tau \in \mathbb{R}^{M}$, be any horizontal lift. Then

$$
\mathcal{H}_{H}^{s}\left(K_{H}(b, \tau)\right)<\infty
$$

where $s$ is the similarity dimension of $\mathcal{A}$. In particular,

$$
\operatorname{dim}_{H} K_{H}(b, \tau) \leqslant s .
$$

Corollary 3.10. If $\left\|A_{i}\right\|<\frac{1}{2}$ for each $i$ and $s \leqslant 2$, then

$$
\operatorname{dim}_{E} K(b)=\operatorname{dim}_{E} K_{H}(b, \tau)=\operatorname{dim}_{H} K_{H}(b, \tau)=s
$$

for a.e. $b \in \mathbb{R}^{2 M}$ and all $\tau \in \mathbb{R}^{M}$.

Proof of Theorem 3.9. Without loss of generality assume that the Heisenberg diameter of $K_{H}(b)$ is 1 .

Since $\mathcal{F}(b)$ consists of similarities of $\mathbb{R}^{2}, \mathcal{F}_{H}(b, \tau)$ consists of similarities of $\mathbb{H}$. The value $r_{i}:=\left\|A_{i}\right\|$ is the common contraction ratio for $f_{i} \in \mathcal{F}(b)$ and its lift $F_{i} \in \mathcal{F}_{H}(b, \tau)$. Denote by $r_{\max }<1$ the maximum of the $r_{i}$.

Given $\delta>0$, choose $m$ so that $r_{\max }^{m}<\delta$. The sets $A_{w}:=F_{w}\left(K_{H}(b, \tau)\right)$, with $w \in W_{m}$, cover $K_{H}(b, \tau)$ and $\operatorname{diam}_{H} A_{w}=r_{w}<\delta$. Thus

$$
\begin{aligned}
\mathcal{H}_{H, \delta}^{s}\left(K_{H}(b, \tau)\right) & \leqslant \sum_{w \in W_{m}}\left(\operatorname{diam}_{H} A_{w}\right)^{s} \\
& =\sum_{w \in W_{m}} r_{w}^{s}=\left(\sum_{i=1}^{M} r_{i}^{s}\right)^{m}=1 .
\end{aligned}
$$

Hence $\mathcal{H}_{H}^{s}\left(K_{H}(b, \tau)\right) \leqslant 1$ and $\operatorname{dim}_{H} K_{H}(b, \tau) \leqslant s$.

REMARK 3.11. The theory developed by Falconer in [10] and recalled in $\S 5$ applies to self-affine systems in arbitrary Euclidean spaces $\mathbb{R}^{n}$. Each self-similar AIFS in $\mathbb{R}^{2}$ lifts to self-similar AIFSs in $\left(\mathbb{H}, d_{H}\right)$ which are not self-similar when viewed as AIFSs on $\left(\mathbb{R}^{3}, d_{E}\right)$. It is an interesting exercise to use Theorem 1.16 to verify that the Euclidean dimension of the lifted fractal agrees with the similarity dimension in this case. 


\section{Comparison of Euclidean and Heisenberg dimensions}

In this section we discuss the application of Theorem 1.10 to the problem of Gromov. In particular, we will prove Theorem 1.7, whose statement we now recall.

TheOREM 4.1. For each $\alpha \in[0,3]$ there exists $S_{\alpha} \subset \mathbb{H}$ with

$$
\mathcal{H}_{E}^{\alpha}\left(S_{\alpha}\right)>0 \quad \text { and } \quad \mathcal{H}_{H}^{\max \{\alpha, 2 \alpha-2\}}\left(S_{\alpha}\right)<\infty \text {. }
$$

Let us also recall that relevant examples for the cases $0 \leqslant \alpha<2$ and $\alpha=3$ of Theorem 4.1 were previously given by Balogh, Rickly and Serra-Cassano [5].

Proof of Theorem 4.1. By Theorem 1.10, each horizontal lift $Q_{H}$ of the unit square serves as the desired example $S_{2}$ in Theorem 4.1 in the case $\alpha=2$. Indeed $\mathcal{H}_{H}^{2}\left(S_{2}\right)<\infty$ while $\mathcal{H}_{E}^{2}\left(S_{2}\right) \geqslant \mathcal{H}_{E}^{2}(Q)=1$.

To treat the case $2<\alpha<3$, we construct certain product-type sets over $Q_{H}$. Let $p=\alpha-2$ and consider a Cantor set $C_{p}$ in the $t$-axis with $0<\mathcal{H}_{E}^{p}\left(C_{p}\right)<\infty$ and $0<\mathcal{H}_{H}^{2 p}\left(C_{p}\right)<\infty$. The construction of such a set is standard; see, for example, $[\mathbf{3}$, p. 300] or [5, $\S 4]$. To wit, choosing $s=2^{-1 / p}$ we view $C_{p}$ as the invariant set associated with the system $\mathcal{G}_{H}=\left\{G_{1}, G_{2}\right\}$, where $G_{1}$ and $G_{2}$ are the $\sqrt{s}$-Lipschitz maps of $\left(\mathbb{H}, d_{H}\right)$ defined by $G_{1}(x, t)=(\sqrt{s} x, s t)$ and $G_{2}(x, t)=(\sqrt{s} x, 1+s(t-1))$.

The set $S_{\alpha}$ is defined as the following product of $Q_{H}$ with $C_{p}$ :

$$
S_{\alpha}:=\left\{\left(x, t+t^{\prime}\right):(x, t) \in Q_{H},\left(0, t^{\prime}\right) \in C_{p}\right\} .
$$

The estimate $\mathcal{H}_{E}^{\alpha}\left(S_{\alpha}\right)=\mathcal{H}_{E}^{2+p}\left(S_{\alpha}\right)>0$ is a consequence of the Euclidean product structure of $S_{\alpha}$, as follows. For $x \in Q$ define

$$
t_{x}=\max \left\{t:(x, t) \in Q_{H}\right\}
$$

and $\Phi: Q \times C_{p} \rightarrow S_{\alpha}$ by

$$
\Phi(x,(0, t))=\left(x, t_{x}+t\right) .
$$

The map $\Phi$ is an expanding (1-co-Lipschitz) embedding of $Q \times C_{p}$ into $S_{\alpha}$. Thus it suffices to show that

$$
\mathcal{H}_{E}^{\alpha}\left(Q \times C_{p}\right)=\mathcal{H}_{E}^{2+p}\left(Q \times C_{p}\right)>0 .
$$

This follows from [19, Theorem 8.10], since $\mathcal{H}_{E}^{2}(Q)=1$ and $\mathcal{H}_{E}^{p}\left(C_{p}\right)>0$.

To show the estimate $\mathcal{H}_{H}^{2 \alpha-2}\left(S_{\alpha}\right)=\mathcal{H}_{H}^{2+2 p}\left(S_{\alpha}\right)<\infty$ we use the obvious covering of $S_{\alpha}$ by similarity images of $Q$ and $C_{p}$. Fix $\delta>0$ and choose

$$
m>\frac{1}{4}+\frac{1}{2 p}+\frac{\log 1 / \delta}{\log 2} .
$$

Set $n=[2 p m]$, where $[x]$ denotes the greatest integer less than or equal to $x$, and consider the covering of $S_{\alpha}$ with the sets

$$
S_{v w}:=\left\{\left(x, t+t^{\prime}\right):(x, t) \in F_{w}\left(Q_{H}\right),\left(0, t^{\prime}\right) \in G_{v}\left(C_{p}\right)\right\},
$$

where $w$ and $v$ range over the sets $W_{m}=\{1,2,3,4\}^{m}$ and $V_{n}=\{1,2\}^{n}$ respectively.

To estimate $\operatorname{diam}_{H}\left(S_{v w}\right)$, choose $\left(x, t+t^{\prime}\right)$ and $\left(\widetilde{x}, \widetilde{t}+\widetilde{t}^{\prime}\right)$ in $S_{v w}$ with

$$
\operatorname{diam}_{H}\left(S_{v w}\right)=d_{H}\left(\left(x, t+t^{\prime}\right),\left(\widetilde{x}, \widetilde{t}+\widetilde{t}^{\prime}\right)\right)
$$


and compute

$$
\begin{aligned}
\operatorname{diam}_{H}\left(S_{v w}\right)^{4} & =|\widetilde{x}-x|^{4}+\left(\widetilde{t}-t+\widetilde{t}^{\prime}-t^{\prime}-2\langle x, J \widetilde{x}\rangle\right)^{2} \\
& \leqslant 2\left(|\widetilde{x}-x|^{4}+(\widetilde{t}-t-2\langle x, J \widetilde{x}\rangle)^{2}+\left(\widetilde{t}^{\prime}-t^{\prime}\right)^{2}\right) \\
& \leqslant 2\left(\left(\frac{1}{2}\right)^{4 m}+\alpha^{2 n}\right)<\delta^{4}
\end{aligned}
$$

Thus

$$
\begin{aligned}
\mathcal{H}_{H, \delta}^{2+2 p}\left(S_{\alpha}\right) & \leqslant \sum_{w \in W_{m}} \sum_{v \in V_{n}} \operatorname{diam}_{H}\left(S_{v w}\right)^{2+2 p} \\
& \leqslant 2^{1 / 4} \cdot 4^{m} \cdot 2^{n} \cdot\left(\left(\frac{1}{2}\right)^{4 m}+\alpha^{2 n}\right)^{(1+p) / 2} \\
& \leqslant C(p) 2^{2 m(1+p)}\left(\left(\frac{1}{2}\right)^{2 m(1+p)}+\alpha^{2 m(1+p) p}\right)=2 C(p)<\infty
\end{aligned}
$$

as desired.

\section{Self-affine horizontal fractals: part I}

In this section, we collect some preliminary material on the Euclidean and Heisenberg critical exponents for a family of linear maps, and Hausdorff-type measures on sequence space defined using these quantities. We also give an example of a Heisenberg AIFS whose dimension can be estimated using our theorems.

\subsection{Singular value functions and critical exponents}

Let $n \geqslant 2$ be an integer. For a contracting linear map $A: \mathbb{R}^{n} \rightarrow \mathbb{R}^{n}$ denote by $1>\alpha_{1} \geqslant \ldots \geqslant \alpha_{n}>0$ the singular values of $A$, defined as the lengths of the principal semi-axes of the ellipsoid $A\left(B^{n}(0,1)\right)$, or equivalently as the positive square roots of the eigenvalues of $A^{T} A$. The singular value function $\varphi^{s}(A)$ is defined for $s \geqslant 0$ as

$$
\varphi^{s}(A)=\alpha_{1} \alpha_{2} \ldots \alpha_{m-1} \alpha_{m}^{s-m+1}, \quad \text { for } 0<s \leqslant n,
$$

where $m$ is the integer such that $m-1<s \leqslant m, \varphi^{0}(A)=1$, and

$$
\varphi^{s}(A)=\left(\alpha_{1} \ldots \alpha_{n}\right)^{s / n}, \text { for } s>n .
$$

Given a collection $\mathcal{A}=\left\{A_{1}, \ldots, A_{M}\right\}$ of linear maps in $\mathbb{R}^{n}$, define the critical exponent $s_{E}(\mathcal{A})$ as the unique non-negative solution $s$ to the equation

$$
\lim _{m \rightarrow \infty}\left(\sum_{w \in W_{m}} \varphi^{s}\left(A_{w}\right)\right)^{1 / m}=1,
$$

where $A_{w}=A_{w_{1}} \ldots A_{w_{m}}$ and $w=w_{1} \ldots w_{m} \in W_{m}:=\{1, \ldots, M\}^{m}$. This critical exponent is the value which appears in the theorem of Falconer and Solomyak from the introduction. If each element of $\mathcal{A}$ is conformal, $s_{E}(\mathcal{A})$ is equal to the similarity dimension of $\mathcal{A}$.

Recall that each horizontal lift of a planar affine map $f(x)=A x+b$ is an affine map $F(x, t)=\widetilde{A}_{b}(x, t)+\widetilde{b}$, where $\widetilde{A}_{b}$ and $\widetilde{b}=(b, \tau)$, with $\tau \in \mathbb{R}$, are defined in (2.2). For fixed $b \in \mathbb{R}^{2 M}$ and a planar AIFS $\mathcal{F}(b)$, we denote by $\mathcal{F}_{H}(b, \tau)$ the lifted IFS corresponding to a specific choice of $\tau \in \mathbb{R}^{M}$ and by $K_{H}(b, \tau)$ its invariant set. Also, denote by $\widetilde{s}_{E}(b ; \mathcal{A})$ the critical exponent for the family $\left\{\widetilde{A}_{1, b_{1}}, \ldots, \widetilde{A}_{M, b_{M}}\right\}$, as defined above, and abbreviate $\widetilde{s}_{E}(\mathcal{A}):=\widetilde{s}_{E}(0 ; \mathcal{A})$.

We now recall the statement of Theorem 1.18 from the introduction. 
ThEOREM 5.3. Let $\mathcal{F}(b)$, with $b \in \mathbb{R}^{2 M}$, be an IFS of affine maps in $\mathbb{R}^{2}$ and let $\mathcal{F}_{H}(b, \tau)$, with $\tau \in \mathbb{R}^{M}$, be any horizontal lift to $\mathbb{H}$ as above. Then

(i) $\operatorname{dim}_{E} K_{H}(b, \tau) \leqslant \widetilde{s}_{E}(\mathcal{A})$ for all $b \in \mathbb{R}^{2 M}$ and $\tau \in \mathbb{R}^{M}$; and

(ii) if $\left\|A_{i}\right\|<\frac{1}{2}$ for all $i$, then $\operatorname{dim}_{E} K_{H}(b, \tau)=\min \left\{3, \widetilde{s}_{E}(\mathcal{A})\right\}$ for a.e. $b \in \mathbb{R}^{2 M}$ and $\tau \in \mathbb{R}^{M}$.

If $\alpha_{1}$ and $\alpha_{2}$ are the singular values of $A$ then the singular values of the $3 \times 3$ matrix $\widetilde{A}_{0}$ are $\alpha_{1}, \alpha_{2}$ and $\alpha_{1} \alpha_{2}$, as can easily be seen from (2.2). It follows that $\widetilde{s}_{E}(\mathcal{A})$ is the unique non-negative solution $s$ to the equation

$$
\lim _{m \rightarrow \infty}\left(\sum_{w \in W_{m}} \widetilde{\varphi}^{s}\left(A_{w}\right)\right)^{1 / m}=1,
$$

where $\widetilde{\varphi}^{s}$ is the modified singular value function

$$
\widetilde{\varphi}^{s}(A)= \begin{cases}\alpha_{1}^{s} & \text { if } 0<s \leqslant 1, \\ \alpha_{1} \alpha_{2}^{s-1} & \text { if } 1<s \leqslant 2, \\ \alpha_{1}^{s-1} \alpha_{2}^{s-1} & \text { if } 2<s \leqslant 3, \\ \alpha_{1}^{2 s / 3} \alpha_{2}^{2 s / 3} & \text { if } 3<s,\end{cases}
$$

and $\widetilde{\varphi}^{0}(A)=1$. Note that $\varphi^{s}=\widetilde{\varphi}^{s}$ for $0 \leqslant s \leqslant 2$.

For $\widetilde{s}_{E}(\mathcal{A}) \leqslant 3$, the estimate

$$
\widetilde{s}_{E}(\mathcal{A}) \leqslant \widetilde{s}_{E}(b ; \mathcal{A})
$$

clearly follows from (1.17) and Theorem 5.3(ii) for a.e. $b \in \mathbb{R}^{2 M}$. In fact, (5.6) holds without restriction. This is a purely linear algebraic fact which can be proved by adapting a theorem of Golub [14] on singular values of rank 1 perturbations of diagonal matrices. See the appendix for details.

Next, for a contracting linear map $A$ of $\mathbb{R}^{2}$ with singular values $1>\alpha_{1} \geqslant \alpha_{2}>0$, define the Heisenberg singular value function $\psi^{s}(A)$, for $0 \leqslant s \leqslant 4$, as

$$
\psi^{s}(A)= \begin{cases}\alpha_{1}^{s} & \text { if } 0<s \leqslant 1 \\ \alpha_{1}^{(s+1) / 2} \alpha_{2}^{(s-1) / 2} & \text { if } 1<s \leqslant 3 \\ \alpha_{1}^{2} \alpha_{2}^{s-2} & \text { if } 3<s \leqslant 4\end{cases}
$$

and $\psi^{0}(A)=1$. Note that $\varphi^{s}=\psi^{s}$ for $0 \leqslant s \leqslant 1$ and $\widetilde{\varphi}^{s} \leqslant \psi^{s}$ for all $0 \leqslant s \leqslant 3$.

Given a family of linear maps $\mathcal{A}=\left\{A_{1}, \ldots, A_{M}\right\}$ on $\mathbb{R}^{2}$, we define the Heisenberg critical exponent $\widetilde{s}_{H}(\mathcal{A})$ as the unique non-negative solution $s$ to the equation

$$
\lim _{m \rightarrow \infty}\left(\sum_{w \in W_{m}} \psi^{s}\left(A_{w}\right)\right)^{1 / m}=1 .
$$

We now restate Theorem 1.19 from the introduction.

TheOREM 5.9. Let $\mathcal{F}(b)$, with $b \in \mathbb{R}^{2 M}$, be an IFS of affine maps in $\mathbb{R}^{2}$ and let $\mathcal{F}_{H}(b, \tau)$, with $\tau \in \mathbb{R}^{M}$, be any horizontal lift to $\mathbb{H}$ as above. Then

(i) $\operatorname{dim}_{H} K_{H}(b, \tau) \leqslant \widetilde{s}_{H}(\mathcal{A})$ for all $b \in \mathbb{R}^{2 M}$ and $\tau \in \mathbb{R}^{M}$; and

(ii) if $\left\|A_{i}\right\|<\frac{1}{2}$ for each $i$, then $\operatorname{dim}_{H} K_{H}(b, \tau)=\min \left\{4, \widetilde{s}_{H}(\mathcal{A})\right\}$ for a.e. $b \in \mathbb{R}^{2 M}$ and $\tau \in \mathbb{R}^{M}$. 
The singular value functions defined in (5.1) and (5.7) may be interpreted as follows. In the Euclidean case, the image of a cube $Q$ of side length 1 in $\mathbb{R}^{n}$ under $A$ is a rectilinear parallelepiped with sides of length $\alpha_{1}, \ldots, \alpha_{n}$. In the singular value function

$$
\varphi^{s}(A)=\frac{\alpha_{1}}{\alpha_{m}} \ldots \frac{\alpha_{m-1}}{\alpha_{m}} \cdot \alpha_{m}^{s},
$$

the term $\left(\alpha_{1} / \alpha_{m}\right) \ldots\left(\alpha_{m-1} / \alpha_{m}\right)$ counts (roughly) the number of cubes $Q^{\prime}$ of side length $\alpha_{m}$ needed to cover $A(Q)$, while the term $\alpha_{m}^{s}$ represents the $s$ th power of the diameter of such a cube $Q^{\prime}$.

In the Heisenberg case, the image of $Q \subset \mathbb{R}^{3}=\mathbb{H}$ under a lift $\widetilde{A}$ of $A$ is a (skewed) parallelepiped, whose base is a rectangle with sides of length $\alpha_{1}$ and $\alpha_{2}$ and which has Euclidean height $\alpha_{1} \alpha_{2}$ and Heisenberg height $\sqrt{\alpha_{1} \alpha_{2}}$. Then, in the singular value function

$$
\psi^{s}(A)= \begin{cases}1 \cdot \alpha_{1}^{s} & \text { if } 0<s \leqslant 1, \\ \frac{\alpha_{1}}{\sqrt{\alpha_{1} \alpha_{2}}}\left(\sqrt{\alpha_{1} \alpha_{2}}\right)^{s} & \text { if } 1<s \leqslant 3, \\ \left(\frac{\alpha_{1}}{\alpha_{2}}\right)^{2} \alpha_{2}^{s} & \text { if } 3<s \leqslant 4,\end{cases}
$$

the terms $1, \alpha_{1} / \sqrt{\alpha_{1} \alpha_{2}}$ and $\left(\alpha_{1} / \alpha_{2}\right)^{2}$ count the number of Heisenberg cubes $Q^{\prime}$ of the appropriate size needed to cover $\widetilde{A}(Q)$, while the final term $\alpha_{1}^{s},\left(\sqrt{\alpha_{1} \alpha_{2}}\right)^{s}$, or $\alpha_{2}^{s}$ represents the $s$ th power of the (Heisenberg) diameter of such a cube $Q^{\prime}$.

\subsection{Measures of Hausdorff type on $\Sigma$}

Fix $s \geqslant 0$. Following Falconer [10, $\S 4$, we define certain measures of Hausdorff type on symbolic space $\Sigma$. A collection $\Lambda$ of finite words is called a partition of $\Sigma$ if $\Sigma$ is the disjoint union of the cylinder sets $\Sigma_{w}$, with $w \in \Lambda$.

Let $\mathcal{A}$ be a finite collection of linear maps in $\mathbb{R}^{n}$, with $n=2,3$. For $m \in \mathbb{N}$ and $S \subset \Sigma$ let

$$
\mathcal{M}_{E, m}^{s}(S):=\inf _{\Lambda} \sum_{\substack{w \in \Lambda \\ S \cap \Sigma_{w} \neq \emptyset}} \varphi^{s}\left(A_{w}\right),
$$

where the infimum is taken over all partitions $\Lambda$ of $\Sigma$ with words of length at least $m$. Next, let

$$
\mathcal{M}_{E}^{s}(S)=\lim _{m \rightarrow \infty} \mathcal{M}_{E, m}^{s}(S)
$$

Then $\mathcal{M}_{E}^{s}$ is an outer measure on $\Sigma$. The Borel subsets of $\Sigma$ are $\mathcal{M}_{E}^{s}$-measurable, so $\mathcal{M}_{E}^{s}$ restricts to a Borel measure on $\Sigma$. The technical term for $\mathcal{M}_{E}^{s}$ is the Method II measure constructed from the premeasure $\tau\left(\Sigma_{w}\right)=\varphi^{s}\left(A_{w}\right)$ on the net $\left\{\Sigma_{w}: w \in W\right\}$. See Rogers [21] for the relevant definitions and vocabulary.

In a similar manner, we define $\widetilde{\mathcal{M}}_{H, m}^{s}$ and $\widetilde{\mathcal{M}}_{H}^{s}$ by replacing $\varphi^{s}$ in the above equation with $\psi^{s}$. Then $\widetilde{\mathcal{M}}_{H}^{s}$ is again a Method II Borel net measure on $\Sigma$.

By Proposition 4.1 of $[\mathbf{1 0}]$, the Euclidean critical exponent $s_{E}(\mathcal{A})$ defined via (5.2) is also equal to

$$
\inf \left\{s: \mathcal{M}_{E}^{s}(\Sigma)=0\right\}=\sup \left\{s: \mathcal{M}_{E}^{s}(\Sigma)=\infty\right\}
$$

In a similar manner, we show the following result. 
Proposition 5.10. The Heisenberg critical exponent $\widetilde{s}_{H}(\mathcal{A})$ defined via $(5.8)$ is equal to

$$
\inf \left\{s: \widetilde{\mathcal{M}}_{H}^{s}(\Sigma)=0\right\}=\sup \left\{s: \widetilde{\mathcal{M}}_{H}^{s}(\Sigma)=\infty\right\}
$$

The proof is completely analogous to the proof of [10, Proposition 4.1] and will be omitted. The relevant features of the singular value function $\psi^{s}$ which are necessary for the proof are:

(i) $\psi^{s}\left(A_{w}\right)$ is submultiplicative in $w: \psi^{s}\left(A_{w w^{\prime}}\right) \leqslant \psi^{s}\left(A_{w}\right) \psi^{s}\left(A_{w^{\prime}}\right)$,

(ii) $\psi^{s}\left(A_{w}\right)$ is decreasing in $s$.

These properties are easily proved using the definition of $\psi^{s}$.

The following technical result on Method II net measures will be used in the proof of Theorem 1.19. The case $\mu=\mathcal{M}_{E}^{s}, \tau\left(\Sigma_{w}\right)=\varphi^{s}\left(A_{w}\right)$ is Lemma 4.2 in [10], but the result holds for any Method II net measure $\mu$ on $\Sigma$ satisfying the assumptions. In particular, it holds for $\mu=\widetilde{\mathcal{M}}_{H}^{s}, \tau\left(\Sigma_{w}\right)=\psi^{s}\left(A_{w}\right)$. Compare Theorem 54 of $[\mathbf{2 1}]$.

LEMMA 5.11. Let $\mu=\sup _{\delta>0} \mu_{\delta}$ be a non-atomic Method II net measure on $\Sigma$ of infinite total mass, defined from a finite premeasure $\tau$ on the cylinder sets $\left\{\Sigma_{w}: w \in W\right\}$. Assume that $\mu_{\delta}\left(C_{j}\right) \rightarrow 0$ as $j \rightarrow \infty$ for every $\delta>0$ and every sequence $C_{1} \supset C_{2} \supset \ldots$ of compact subsets of $\Sigma$ with $\mu\left(\bigcap_{j} C_{j}\right)=0$.

Then there exists a compact subset $C_{0} \subset \Sigma$ so that $0<\mu\left(C_{0}\right)<\infty$ and there exists a constant $C<\infty$ so that

$$
\mu\left(C_{0} \cap \Sigma_{w}\right) \leqslant C \tau\left(\Sigma_{w}\right)
$$

for all $w \in W$.

The following example shows that the second inequality in (3.1) can be strict for self-affine fractals.

EXAMPLE 5.13. Fix integers $n \geqslant p \geqslant 2$ and consider the planar AIFS $\mathcal{F}=\left\{f_{11}, \ldots, f_{n p}\right\}$, where $f_{i j}\left(x_{1}, x_{2}\right)=\left(\left(x_{1}+i\right) / n,\left(x_{2}+j\right) / p\right)$, for $i=1, \ldots, n$ and $j=1, \ldots, p$. The invariant set for $\mathcal{F}$ is the unit square $Q=[0,1]^{2}$, viewed as the selfaffine set obtained by gluing together $n p$ rectangles with sides of length $1 / n$ and $1 / p$. In this case

$$
A_{i j}=\left(\begin{array}{cc}
1 / n & 0 \\
0 & 1 / p
\end{array}\right) \text { and } b_{i j}=\left(\begin{array}{c}
i / n \\
j / p
\end{array}\right) .
$$

For $w \in W_{m}=\{1, \ldots, n p\}^{m}$, the singular values of $A_{w}$ are $p^{-m}$ and $n^{-m}$. Then

$$
\lim _{m \rightarrow \infty}\left(\sum_{w \in W_{m}} \psi^{s}\left(A_{w}\right)\right)^{1 / m}= \begin{cases}n p^{1-s} & \text { if } 0 \leqslant s \leqslant 1 \\ n^{(3-s) / 2} p^{(1-s) / 2} & \text { if } 1 \leqslant s \leqslant 3 \\ n^{3-s} p^{-1} & \text { if } 3 \leqslant s \leqslant 4\end{cases}
$$

Thus

$$
\operatorname{dim}_{H} K_{H}(\tau) \leqslant \widetilde{s}_{H}(\mathcal{A})=1+\frac{2 \log n}{\log (n p)}
$$

for any Heisenberg lift $\mathcal{F}_{H}(\tau)$ of $\mathcal{F}$. Note that $\widetilde{s}_{H}(\mathcal{A})=2$ only in the self-similar case $n=p$.

From $(5.5)$ it easily follows that $\tilde{s}_{E}(\mathcal{A})=s_{E}(\mathcal{A})=2$. Thus

$$
\operatorname{dim}_{E} K_{H}(\tau)=2
$$

for all $\tau$. 
REMARK 5.14. In a subsequent paper [11], Falconer derived lower bounds for $\operatorname{dim}_{E} K(b)$ which hold for every $b$. Let $s_{-}=s_{-}\left(A_{1}, \ldots, A_{M}\right)$ be the unique non-negative solution to the equation

$$
\lim _{m \rightarrow \infty}\left(\sum_{w \in W_{m}} \varphi^{s}\left(A_{w}^{-1}\right)^{-1}\right)^{1 / m}=1 .
$$

Then [11, Proposition 2] reads as follows.

Proposition 5.16. If $\mathcal{F}$ satisfies the disjointness condition

$$
f_{i}(K(b)) \cap f_{j}(K(b))=\emptyset \quad \text { for every } i \neq j,
$$

then

$$
\operatorname{dim}_{E} K(b) \geqslant s_{-} .
$$

Note that the open set condition does not suffice to imply (5.17); see [11, Example 2] for an example of an AIFS $\mathcal{F}$ in $\mathbb{R}^{2}$ such that $s_{-}>0$ but $K(b)$ is a single point.

The claim regarding the Euclidean dimension of the horizontal lift in Example 3.2 may be proved using Proposition 5.16.

\section{Self-affine horizontal fractals: part II}

In this section, we give the proofs of Theorems 1.18 and 1.19. To simplify the exposition, we will present the proofs of the first parts of both theorems together, followed by the proofs of the second parts. In each case, we present in detail the proof for the Heisenberg dimension (Theorem 1.19) and only sketch how this proof should be modified for the Euclidean dimension (Theorem 1.18).

Proof of Theorem 1.19(i). Fix $b \in \mathbb{R}^{2 M}, \tau \in \mathbb{R}^{M}$ and $s>\widetilde{s}_{H}(\mathcal{A})$. We will show that

$$
\mathcal{H}_{H}^{s}\left(K_{H}(b, \tau)\right) \leqslant C \widetilde{\mathcal{M}}_{H}^{s}(\Sigma)
$$

for some absolute constant $C$. Since $\widetilde{\mathcal{M}_{H}^{s}}(\Sigma)=0$ by Proposition 5.10 , this suffices to complete the proof.

Let $0<\alpha<1$ be so that

$$
d_{H}\left(F_{i}(p), F_{i}(q)\right)<\alpha d_{H}(p, q)
$$

for $p, q \in \mathbb{H}$ and $i=1, \ldots, M$. Let $B=B_{H}(0, R) \subset \mathbb{H}$ be a Heisenberg ball centered at the origin of radius $R$, chosen so large that $F_{i}(B) \subset B$ for all $i$. Given $\delta>0$, choose $m$ so large that $\alpha^{m}<\delta$.

Let $\Lambda$ be an arbitrary partition of $\Sigma$ by words of length at least $m$. By the choice of $m, \operatorname{diam}_{H} F_{w}(B)<\delta$ for all $w \in \Lambda$. For each $w \in \Lambda$, we may write $F_{w}(x, t)=\widetilde{A}_{w, b_{w}}(x, t)+\widetilde{b}_{w}$, where $\widetilde{A}_{w, b_{w}}$ and $\widetilde{b}_{w}$ are given by the formulas in (2.2). If we denote by $\alpha_{i, 1} \geqslant \alpha_{i, 2}$ the singular values of $A_{i}$, for $i=1, \ldots, M$, then the singular values of $A_{w}$ are $\alpha_{w, 1} \geqslant \alpha_{w, 2}$, where

$$
\alpha_{w, j} \leqslant \prod_{i=1}^{m} \alpha_{w_{i}, j} \leqslant \alpha^{m}
$$

for any word $w$ of length $m$. 
In what follows we fix a word $w$ and write $\alpha_{j}=\alpha_{w, j}$, for $j=1,2$. Let $Q_{w}$ be a rectangle containing $\pi\left(F_{w}(B)\right)=f_{w}(\pi(B))$ with sides of length $3 R \alpha_{1}$ and $3 R \alpha_{2}$. Observe that $b_{w}=f_{w}(0) \in Q_{w}$. Then $F_{w}(B) \subset \widetilde{Q}_{w}$, where

$$
\widetilde{Q}_{w}=\left\{(x, t): x \in Q_{w},\left|t+2\left\langle x, J b_{w}\right\rangle-\tau_{w}\right|<R^{2} \alpha_{1} \alpha_{2}\right\}
$$

is a parallelepiped with base $Q_{w} \in \mathbb{R}^{2}$ and (Euclidean) height $2 R^{2} \alpha_{1} \alpha_{2}$.

If $(x, t)$ and $\left(x^{\prime}, t^{\prime}\right)$ are elements of $\widetilde{Q}_{w}$, then

$$
\left|x^{\prime}-x\right| \leqslant \operatorname{diam} Q_{w} \leqslant 3 \sqrt{2} R \alpha_{1}
$$

and

$$
\left|t^{\prime}-t-2\left\langle x, J x^{\prime}\right\rangle\right| \leqslant 2 R^{2} \alpha_{1} \alpha_{2}+2\left|\left\langle x^{\prime}-x, J\left(b_{w}-x\right)\right\rangle\right| .
$$

We distinguish three cases according to the value of $s$.

Case 1: $0 \leqslant s \leqslant 1$. From (6.2) we have $\left|x-x^{\prime}\right| \leqslant C R \alpha_{1}$. Since $b_{w} \in Q_{w}$ we obtain likewise that $\left|b_{w}-x\right| \leqslant C R \alpha_{1}$. Using (6.3) we deduce that

$$
\left|t^{\prime}-t-2\left\langle x, J x^{\prime}\right\rangle\right| \leqslant C R^{2} \alpha_{1}^{2} .
$$

Using this and (6.2) in (1.2) we obtain

$$
\operatorname{diam}_{H} \widetilde{Q}_{w} \leqslant C R \alpha_{1}
$$

The sets $\widetilde{Q}_{w}$, with $w \in \Lambda$, cover $K_{H}(b, \tau)$ and we obtain

$$
\mathcal{H}_{H, C R \delta}^{s}\left(K_{H}(b, \tau)\right) \leqslant C(R, s) \sum_{w \in \Lambda} \alpha_{1}^{s}=C(R, s) \sum_{w \in \Lambda} \psi^{s}\left(A_{w}\right)
$$

by the choice of $m$.

Observe that in this estimate the dependence of $b$ does not appear at all; this will also happen in the other cases.

Case 2: $1 \leqslant s \leqslant 3$. In this case we divide $\widetilde{Q}_{w}$ into at most $K:=2 \alpha_{1} / \sqrt{\alpha_{1} \alpha_{2}}$ smaller parallelepipeds $\widetilde{P}_{j}$ whose base is a rectangle $P_{j}$ in $\mathbb{R}^{2}$ with sides of length $3 R \sqrt{\alpha_{1} \alpha_{2}}$ and $3 R \alpha_{2}$ and whose (Euclidean) height is still $2 R^{2} \alpha_{1} \alpha_{2}$.

Our task is to estimate the Heisenberg diameter of such a parallelepiped $\widetilde{P}_{j}$. Let $(x, t)$ and $\left(x^{\prime}, t^{\prime}\right)$ be elements of $\widetilde{P}_{j}$. Then

$$
\left|x^{\prime}-x\right| \leqslant 3 \sqrt{2} R \sqrt{\alpha_{1} \alpha_{2}}
$$

and (by $(6.3))$

$$
\left|t^{\prime}-t-2\left\langle x, J x^{\prime}\right\rangle\right| \leqslant 2 R^{2} \alpha_{1} \alpha_{2}+2\left|\left\langle x^{\prime}-x, J\left(b_{w}-x\right)\right\rangle\right| .
$$

The expression $\left|\left\langle x^{\prime}-x, J\left(b_{w}-x\right)\right\rangle\right|$ equals twice the area of the planar triangle with vertices $x^{\prime}, x, b_{w}$ which lies in $Q_{w}$. This yields

$$
\left|\left\langle x^{\prime}-x, J\left(b_{w}-x\right)\right\rangle\right| \leqslant 2 \alpha_{1} \alpha_{2}
$$

and so we have

$$
\operatorname{diam}_{H} \widetilde{P}_{j} \leqslant C R \sqrt{\alpha_{1} \alpha_{2}}
$$

using (1.2). 
The sets $\widetilde{P}_{j}$, for $j=1, \ldots, K$, associated with each $\widetilde{Q}_{w}$, where $w \in \Lambda$, cover $K_{H}(b, \tau)$ and we obtain

$$
\mathcal{H}_{H, C R \delta}^{s}\left(K_{H}(b, \tau)\right) \leqslant C(R, s) \sum_{w \in \Lambda} \sum_{j=1}^{K}\left(\sqrt{\alpha_{1} \alpha_{2}}\right)^{s} \leqslant C(R, s) \sum_{w \in \Lambda} \psi^{s}\left(A_{w}\right)
$$

by the choices of $m$ and $K$.

Case 3: $3 \leqslant s \leqslant 4$. As in the previous case we begin by dividing $\widetilde{Q}_{w}$ into at most $N:=2 \alpha_{1} / \alpha_{2}$ smaller parallelepipeds $\widetilde{P}_{j}$ whose base is a square $P_{j}$ in $\mathbb{R}^{2}$ with side length $3 R \alpha_{2}$ and whose (Euclidean) height is at most $40 R^{2} \alpha_{1} \alpha_{2}$. Explicitly, let $c_{w j}$ be the center of the square $P_{j}$ and let $\widetilde{P}_{j}$ be the set of points $(x, t) \in \widetilde{Q}_{w}$ for which $x \in P_{j}$ and

$$
\left|t+2\left\langle x-b_{w}, J c_{w j}\right\rangle-\tau_{w}\right|<40 R^{2} \alpha_{1} \alpha_{2}
$$

Observe that

$$
\widetilde{Q}_{w} \subset \bigcup_{j=1}^{N} \widetilde{P}_{j}
$$

Indeed, $(x, t) \in \widetilde{Q}_{w}$ and $x \in P_{j}$ imply that

$$
\begin{aligned}
\left|t+2\left\langle x-b_{w}, J c_{w j}\right\rangle-\tau_{w}\right| & \leqslant\left|t+2\left\langle x, J b_{w}\right\rangle-\tau_{w}\right|+2\left|\left\langle x-b_{w}, J\left(c_{w j}-x\right)\right\rangle\right| \\
& \leqslant R^{2} \alpha_{1} \alpha_{2}+2\left(3 \sqrt{2} R \alpha_{1}\right)\left(3 \sqrt{2} R \alpha_{2}\right) \\
& <40 R^{2} \alpha_{1} \alpha_{2} .
\end{aligned}
$$

Next we show that we can cover $\widetilde{P}_{j}$ by at most $2 N$ Heisenberg balls of the form $B_{H}\left(p_{j k}, C R \alpha_{2}\right)$ with centers

$$
p_{j k}=\left(c_{w j}, t_{j k}\right)
$$

where

$$
t_{j k}=\tau_{w}+2\left\langle b_{w}, J c_{w j}\right\rangle+20 k R^{2} \alpha_{2}^{2},
$$

for $k=-N, \ldots, N$. Indeed, if $(x, t) \in \widetilde{P}_{j}$ then $x \in P_{j}$ and so $\left|x-c_{w j}\right| \leqslant C R \alpha_{2}$. By (6.4) there exists an integer $k \in[-N, N]$ such that

$$
20 \cdot(k-1) R^{2} \alpha_{2}^{2} \leqslant t+2\left\langle x-b_{w}, J w_{j}\right\rangle-\tau_{w} \leqslant 20 \cdot k R^{2} \alpha_{2}^{2} .
$$

Using (6.5) this implies that for large enough $C>0$ we have

$$
\left|t-t_{j k}+2\left\langle x-c_{w j}, J c_{w j}\right\rangle\right| \leqslant C R^{2} \alpha_{2}^{2}
$$

and so

$$
\left|t-t_{j k}+2\left\langle x, J c_{w j}\right\rangle\right| \leqslant C R^{2} \alpha_{2}^{2} .
$$

From (1.2) we deduce that

$$
d_{H}\left((x, t),\left(c_{w j}, t_{j k}\right)\right) \leqslant C R \alpha_{2},
$$

as required.

The balls $B_{H}\left(p_{j k}, C R \alpha_{2}\right)$, for $j=1, \ldots, N$ and $k=-N, \ldots, N$, associated with each $\widetilde{Q}_{w}$, with $w \in \Lambda$, cover $K_{H}(b, \tau)$ and we obtain

$$
\begin{aligned}
\mathcal{H}_{H, C R \delta}^{s}\left(K_{H}(b, \tau)\right) & \leqslant C(R, s) \sum_{w \in \Lambda} \sum_{j=1}^{N} \sum_{k=-N}^{N} \alpha_{2}^{s} \\
& \leqslant C(R, s) \sum_{w \in \Lambda} \psi^{s}\left(A_{w}\right)
\end{aligned}
$$

by the choices of $m$ and $N$. 
In all cases, we have shown that

$$
\mathcal{H}_{H, C R \delta}^{s}\left(K_{H}(b, \tau)\right) \leqslant C(R, s) \sum_{w \in \Lambda} \psi^{s}\left(A_{w}\right) .
$$

Taking the infimum over partitions $\Lambda$ followed by the limit as $m \rightarrow \infty$ yields (6.1). This completes the proof of Theorem 1.19(i).

Proof of Theorem 1.18(i). For this only a few modifications need to be made in the above reasoning. In Case 1 , the Euclidean diameter of $\widetilde{Q}_{w}$ is at most $C R \alpha_{1}$ and so

$$
\mathcal{H}_{E, C R \delta}^{s}\left(K_{H}(b, \tau)\right) \leqslant C(R, s) \sum_{w \in \Lambda} \alpha_{1}^{s} \leqslant C(R, s) \sum_{w \in \Lambda} \varphi^{s}\left(\widetilde{A}_{w, b_{w}}\right)
$$

Case 2 must be split into two subcases: either $1 \leqslant s \leqslant 2$ or $2 \leqslant s \leqslant 3$. In the former case we divide $\widetilde{Q}_{w}$ into at most $N=2 \alpha_{1} / \alpha_{2}$ parallelepipeds whose base is a square of side length $3 R \alpha_{2}$ and estimate

$$
\mathcal{H}_{E, C R \delta}^{s}\left(K_{H}(b, \tau)\right) \leqslant C(R, s) \sum_{w \in \Lambda} \sum_{j=1}^{N} \alpha_{2}^{s} \leqslant C(R, s) \sum_{w \in \Lambda} \varphi^{s}\left(\widetilde{A}_{w, b_{w}}\right) .
$$

In the latter case we divide $\widetilde{Q}_{w}$ into at most $P=2 / \alpha_{1} \alpha_{2}$ parallelepipeds whose base is a square of side length $3 R \alpha_{1} \alpha_{2}$ and estimate

$$
\mathcal{H}_{E, C R \delta}^{s}\left(K_{H}(b, \tau)\right) \leqslant C(R, s) \sum_{w \in \Lambda} \sum_{j=1}^{P}\left(\alpha_{1} \alpha_{2}\right)^{s} \leqslant C(R, s) \sum_{w \in \Lambda} \varphi^{s}\left(\widetilde{A}_{w, b_{w}}\right) .
$$

As before, these estimates suffice to complete the proof of Theorem 1.18(i).

As is typical with problems involving the computation of Hausdorff dimension, obtaining lower bounds is more difficult. Following the technique employed by Falconer in the Euclidean case (Theorem 1.16), we use potential-theoretic arguments to obtain almost sure lower bounds.

We begin with a simple lemma which provides a geometric interpretation of the Heisenberg singular value function. Compare Lemma 2.2 of [10].

Lemma 6.6. Let $0<s<4$, with $s \neq 1,3$. For each $R>0$ there exists a constant $C$ depending only on $R$ and $s$ so that

$$
\int_{B_{H}(0, R)} \frac{d p}{\left|\widetilde{A}_{b}(p)\right|_{H}^{s}} \leqslant \frac{C}{\psi^{s}(A)},
$$

where, for $p \in \mathbb{H}, B_{H}(p, r)$ denotes the ball in the Heisenberg metric $d_{H}$ of radius $r$ and $|p|_{H}=d_{H}(p, 0)$.

In the proof of Lemma 6.6 the following fact (whose proof is an easy exercise) will be used several times.

LEMma 6.8. Let $F: \mathbb{R} \rightarrow \mathbb{R}$ be an even function which is decreasing for $\tau>0$. Let $c \in \mathbb{R}$ and $h>0$. Then $\int_{c-h}^{c+h} F(\tau) d \tau \leqslant \int_{-h}^{h} F(\tau) d \tau$. 
Proof of Lemma 6.6. From the expression for $\widetilde{A}_{b}$ in $(2.2)$ we observe that the integrand in (6.7) equals

$$
\left(|A x|^{4}+(t \operatorname{det} A-2\langle A x, J b\rangle)^{2}\right)^{-s / 4},
$$

where $p=(x, t)$. Choose coordinates in $\mathbb{R}^{2}$ so that $|A x|^{2}=\alpha_{1}^{2} x_{1}^{2}+\alpha_{2}^{2} x_{2}^{2}$. Writing $b=(p, q)$ in these coordinates, we express (6.9) in the form

$$
\left(\left(\alpha_{1}^{2} x_{1}^{2}+\alpha_{2}^{2} x_{2}^{2}\right)^{2}+\left(\alpha_{1} \alpha_{2} t+2 \alpha_{1} x_{1} q-2 \alpha_{2} x_{2} p\right)^{2}\right)^{-s / 4} .
$$

Make the change of variables $y_{i}=\alpha_{i} x_{i} / R$, for $i=1,2$, and

$$
\tau=\frac{\alpha_{1} \alpha_{2} t+2 \alpha_{1} x_{1} q-2 \alpha_{2} x_{2} p}{R^{2}}
$$

in the integral in (6.7) to obtain

$$
\frac{R^{4-s}}{\alpha_{1}^{2} \alpha_{2}^{2}} \int_{E} \int_{I_{y}} \frac{d \tau d y}{\left(|y|^{4}+\tau^{2}\right)^{s / 4}},
$$

where $E=\left\{y:\left(y_{1} / \alpha_{1}\right)^{2}+\left(y_{2} / \alpha_{2}\right)^{2}<1\right\}$,

$$
I_{y}=\left\{\tau:\left|\tau+u y_{2}-v y_{1}\right|<\alpha_{1} \alpha_{2}\right\}
$$

and $(u, v)=(2 / R)(p, q)$.

As before we distinguish three cases according to the value of $s$.

Case 1: $0<s<1$. The integral in (6.10) may be estimated from above by

$$
\frac{C(R, s)}{\alpha_{1}^{2} \alpha_{2}^{2}}\left(\alpha_{2}\right)\left(\alpha_{1} \alpha_{2}\right) \int_{0}^{\alpha_{1}} \frac{d y_{1}}{y_{1}^{s}}=\frac{C(R, s)}{\psi^{s}(A)} .
$$

Case 2: $1<s<3$. The region of integration in (6.10) may be written as $\bigcup_{y \in E} I_{y}=P_{1} \cup P_{2}$, where

$$
P_{1}=\left\{(y, \tau) \in \bigcup_{y \in E} I_{y}: y_{1}^{4}+\left(\tau+u y_{2}-v y_{1}\right)^{2} \leqslant 2 \alpha_{1}^{2} \alpha_{2}^{2}\right\}
$$

and

$$
P_{2}=\left\{(y, \tau) \in \bigcup_{y \in E} I_{y}: y_{1}>\sqrt{\alpha_{1} \alpha_{2}}\right\} .
$$

Write the integral in (6.10) in the form $\left(R^{4-s} \alpha_{1}^{-2} \alpha_{2}^{-2}\right)\left(I_{1}+I_{2}\right)$, where

$$
I_{j}=\int_{P_{j}}\left(|y|^{4}+\tau^{2}\right)^{-s / 4} d y d \tau \text { for } j=1,2 .
$$

For the first term, we use Lemma 6.8 to estimate

$$
\begin{aligned}
I_{1} & \leqslant \int_{-2 \sqrt{\alpha_{1} \alpha_{2}}}^{2 \sqrt{\alpha_{1} \alpha_{2}}} d y_{1} \int_{-\alpha_{2}}^{\alpha_{2}} d y_{2} \int_{-\alpha_{1} \alpha_{2}}^{\alpha_{1} \alpha_{2}} d \tau\left(y_{1}^{4}+\tau^{2}\right)^{-s / 4} \\
& \leqslant C \alpha_{2} \int_{0}^{2 \sqrt{\alpha_{1} \alpha_{2}}} d y_{1} \int_{0}^{\alpha_{1} \alpha_{2}} d \tau\left(y_{1}^{4}+\tau^{2}\right)^{-s / 4} .
\end{aligned}
$$

(Note that $\left|y_{1}\right| \leqslant 2 \sqrt{\alpha_{1} \alpha_{2}}$ for any $(y, \tau) \in P_{1}$.)

Making the Heisenberg change of variables $y_{1}=r \sqrt{\cos \phi}, \tau=r^{2} \sin \phi$ (cf. the polar coordinates in $[6]$ ) we find that

$$
I_{1} \leqslant C \alpha_{2} \int_{0}^{2 \sqrt{\alpha_{1} \alpha_{2}}} r^{2-s} d r=C \alpha_{1}^{(3-s) / 2} \alpha_{2}^{(5-s) / 2} .
$$


Similarly, we obtain the estimate

$$
I_{2} \leqslant C \alpha_{2}\left(\alpha_{1} \alpha_{2}\right) \int_{\sqrt{\alpha_{1} \alpha_{2}}}^{\infty} y_{1}^{-s} d y_{1}=C \alpha_{1}^{(3-s) / 2} \alpha_{2}^{(5-s) / 2} .
$$

Returning to (6.10) we see that

$$
\frac{R^{4-s}}{\alpha_{1}^{2} \alpha_{2}^{2}} \int_{E} \int_{I_{y}} \frac{d \tau d y}{\left(|y|^{4}+\tau^{2}\right)^{s / 4}} \leqslant \frac{C(R, s)}{\psi^{s}(A)}
$$

as desired.

Case 3: $3<s<4$. This is similar to the previous case. We write $\bigcup_{y \in E} I_{y}=$ $P_{1} \cup P_{2}$, where

$$
P_{1}=\left\{(y, \tau) \in \bigcup_{y \in E} I_{y}:|y|^{4}+\left(\tau+u y_{2}-v y_{1}\right)^{2} \leqslant 4 \alpha_{2}^{4}\right\}
$$

and

$$
P_{2}=\left\{(y, \tau) \in \bigcup_{y \in E} I_{y}: y_{1}^{4}+\left(\tau+u y_{2}-v y_{1}\right)^{2}>\alpha_{2}^{4}\right\},
$$

and decompose the integral in (6.10) as before into $I_{1}$ and $I_{2}$ terms. In this case, another application of Lemma 6.8 gives

$$
I_{1} \leqslant \int_{-2 \alpha_{2}}^{2 \alpha_{2}} d y_{1} \int_{-2 \alpha_{2}}^{2 \alpha_{2}} d y_{2} \int_{-2 \alpha_{2}}^{2 \alpha_{2}} d \tau\left(|y|^{4}+\tau^{2}\right)^{-s / 4},
$$

and making the change of variables for integration in Heisenberg polar coordinates $y_{1}=r \sqrt{\cos \phi} \cos \theta, y_{2}=r \sqrt{\cos \phi} \sin \theta, \tau=r^{2} \sin \phi$ yields

$$
I_{1} \leqslant C \int_{0}^{2 \alpha_{2}} r^{3-s} d r=C \alpha_{2}^{4-s}
$$

In a similar manner we obtain the estimate

$$
I_{2} \leqslant C \alpha_{2} \int_{\alpha_{2}}^{\infty} r^{2-s} d r=C \alpha_{2}^{4-s}
$$

and hence

$$
\frac{R^{4-s}}{\alpha_{1}^{2} \alpha_{2}^{2}} \int_{E} \int_{I_{y}} \frac{d y d \tau}{\left(|y|^{4}+\tau^{2}\right)^{s / 4}} \leqslant \frac{C(R, s)}{\psi^{s}(A)}
$$

as desired.

Next, we consider products of matrices indexed by words in $\Sigma$. For a planar AIFS $\mathcal{F}(b)$ with horizontal lift $\mathcal{F}_{H}(\widetilde{b})$ we write $p_{H}(\widetilde{b}): \Sigma \rightarrow K_{H}(\widetilde{b})$ and $p_{E}(b): \Sigma \rightarrow K(b)$ for the canonical surjections from $\Sigma$ to the invariant sets. Thus

$$
p_{H}(\widetilde{b}, w)=\bigcap_{m=1}^{\infty} F_{w_{1}} \circ \ldots \circ F_{w_{m}}\left(K_{H}(\widetilde{b})\right)
$$

and

$$
p_{E}(b, w)=\bigcap_{m=1}^{\infty} f_{w_{1}} \circ \ldots \circ f_{w_{m}}(K(b)),
$$

where $w=w_{1} w_{2} \ldots \in \Sigma$ and $f_{i}(x)=A_{i} x+b_{i}$ and $F_{i}(x, t)=\widetilde{A}_{i, b_{i}}(x, t)+\widetilde{b}_{i}$. 
Observe that

$$
p_{H}(\widetilde{b}, w)=\widetilde{b}_{w_{1}}+\widetilde{A}_{w_{1}, b_{w_{1}}} \cdot \widetilde{b}_{w_{2}}+\widetilde{A}_{w_{1}, b_{w_{1}}} \cdot \widetilde{A}_{w_{2}, b_{w_{2}}} \cdot \widetilde{b}_{w_{3}}+\ldots
$$

For $w$ and $w^{\prime}$ in $\Sigma$ denote by $w \wedge w^{\prime}$ the maximal finite word which is a subword of both $w$ and $w^{\prime}$.

Lemma 6.12. Assume that $\left\|A_{i}\right\|<\frac{1}{2}$ for each $i$. For $0<s<4$, with $s \neq 1,3$, and $R>0$ there exists a constant $C=C(R, s)$ so that

$$
\int_{B_{H}(0, R)^{M}} \frac{d \widetilde{b}}{d_{H}\left(p_{H}(\widetilde{b}, w), p_{H}\left(\widetilde{b}, w^{\prime}\right)\right)^{s}} \leqslant \frac{C}{\psi^{s}\left(A_{w \wedge w^{\prime}}\right)}
$$

for all $w, w^{\prime} \in \Sigma$. Here $\quad B_{H}(0, R)^{M}=B_{H}(0, R) \times \ldots \times B_{H}(0, R) \subseteq \mathbb{R}^{3 M} \quad$ and $\widetilde{b}=\widetilde{b}_{1}, \ldots, \widetilde{b}_{2} \in \mathbb{R}^{3 M}$.

Proof. Write $w \wedge w^{\prime}=\alpha \in W$ and set $w=\alpha v$ and $w^{\prime}=\alpha v^{\prime}$, for $v, v^{\prime} \in \Sigma$. Then

$$
\begin{gathered}
\int_{B_{H}(0, R)^{M}} \frac{d \widetilde{b}}{d_{H}\left(p_{H}(\widetilde{b}, w), p_{H}\left(\widetilde{b}, w^{\prime}\right)\right)^{s}} \\
\quad=\int_{B_{H}(0, R)^{M} \mid} \frac{d \widetilde{b}}{\left.\widetilde{A}_{\alpha, b_{\alpha}}\left(p_{H}(\widetilde{b}, v)^{-1} * p_{H}\left(\widetilde{b}, v^{\prime}\right)\right)\right|_{H} ^{s}} .
\end{gathered}
$$

By the choice of $\alpha, v_{1} \neq v_{1}^{\prime}$. Without loss of generality we may assume that $v_{1}=2$ and $v_{1}^{\prime}=1$.

With (6.11) in mind we make the change of variable

$$
\begin{aligned}
q & =p_{H}(\widetilde{b}, v)^{-1} * p_{H}\left(\widetilde{b}, v^{\prime}\right)=\left(\begin{array}{c}
b_{1}-b_{2}+E(b) \\
\tau_{1}-\tau_{2}+F(\tau)+G(b)
\end{array}\right), \\
\widetilde{b}_{2} & =\widetilde{b}_{2}, \\
& \vdots \\
\widetilde{b}_{M} & =\widetilde{b}_{M},
\end{aligned}
$$

where $E: \mathbb{R}^{2 M} \rightarrow \mathbb{R}^{2}, E(b)=E_{1}\left(b_{1}\right)+\ldots+E_{M}\left(b_{M}\right)$ and $F: \mathbb{R}^{M} \rightarrow \mathbb{R}$ are linear maps and $G: \mathbb{R}^{2 M} \rightarrow \mathbb{R}$ is a quadratic map.

We claim that

$$
\left\|E_{\nu}\right\|<1
$$

for some $\nu=1,2$ and

$$
\|F\|<1 \text {. }
$$

Taking (6.15) and (6.16) for granted observe that the preceding change of variables is invertible. Consequently, we obtain

by Lemma 6.6.

$$
\begin{aligned}
\int_{B_{H}(0, R)^{M}} & \frac{d \widetilde{b}}{d_{H}\left(p_{H}(\widetilde{b}, w), p_{H}\left(\widetilde{b}, w^{\prime}\right)\right)^{s}} \\
& \leqslant C \int_{B_{H}(0,(2+M) R)} d q \int_{B_{H}(0, R)^{M-1}} d \widetilde{b}_{2} \ldots d \widetilde{b}_{M} \frac{1}{\left|\widetilde{A}_{\alpha, b_{\alpha}}(q)\right|_{H}^{s}} \\
& \leqslant \frac{C(R, s)}{\psi^{s}\left(A_{\alpha}\right)}
\end{aligned}
$$


It remains to describe the maps $E, F$ and $G$ explicitly and show (6.15) and (6.16).

Using (6.11) we find that a direct computation yields

$$
p_{H}(\widetilde{b}, v)^{-1} * p_{H}\left(\widetilde{b}, v^{\prime}\right)=\left(\begin{array}{c}
X \\
T
\end{array}\right)
$$

where

$$
\begin{aligned}
X & =p_{E}\left(b, v^{\prime}\right)-p_{E}(b, v) \\
& =b_{v_{1}^{\prime}}-b_{v_{1}}+\left(A_{v_{1}^{\prime}} b_{2}^{\prime}-A_{v_{1}} b_{v_{2}}+A_{v_{1}^{\prime}} A_{v_{2}^{\prime}} b_{v_{3}^{\prime}}-A_{v_{1}} A_{v_{2}} b_{v_{3}}+\ldots\right)
\end{aligned}
$$

(see equation $(3.7)$ in $[\mathbf{1 0}]$ ) and

$$
\begin{aligned}
T= & \left(\tau_{v_{1}^{\prime}}-\tau_{v_{1}}\right)+\left(\lambda_{v_{1}^{\prime}} \tau_{v_{2}^{\prime}}-\lambda_{v_{1}} \tau_{v_{2}}\right)+\left(\lambda_{v_{1}^{\prime}} \lambda_{v_{2}^{\prime}} \tau_{v_{3}^{\prime}}-\lambda_{v_{1}} \lambda_{v_{2}} \tau_{v_{3}}\right)+\ldots \\
& -2\left(\left\langle A_{v_{1}^{\prime}} b_{v_{2}^{\prime}}, J b_{v_{1}^{\prime}}\right\rangle-\left\langle A_{v_{1}} b_{v_{2}}, J b_{v_{1}}\right\rangle+\ldots\right) \\
& -2\left(\left\langle b_{v_{1}^{\prime}}, J b_{v_{1}}\right\rangle+\left\langle A_{v_{1}^{\prime}} b_{v_{2}^{\prime}}, J b_{v_{1}}\right\rangle+\left\langle b_{v_{1}^{\prime}}, J A_{v_{1}} b_{v_{2}}\right\rangle+\ldots\right),
\end{aligned}
$$

where $\lambda_{v_{i}}=\operatorname{det} A_{i}$. Observe that the last term in equation (6.18) is the contribution to $T$ from the term $-2\left\langle p_{E}(\widetilde{b}, v), J p_{E}\left(\widetilde{b}, v^{\prime}\right)\right\rangle$.

We may choose $\nu$ equal to either 1 or 2 and an index $m$ with $2 \leqslant m \leqslant \infty$ so that the following conditions hold:

(i) for each $k<m$, both $v_{k}$ and $v_{k}^{\prime}$ are not equal to $\nu$, and

(ii) if $m<\infty$, then $v_{m} \neq \nu$ and $v_{m}^{\prime} \neq \nu$.

From (6.17) and (6.18) we have

$$
X=b_{1}-b_{2}+E(b)=b_{1}-b_{2}+E_{1}\left(b_{1}\right)+\ldots+E_{M}\left(b_{M}\right)
$$

and

$$
T=\tau_{1}-\tau_{2}+F(\tau)+G(b),
$$

where the $E_{i}$ are linear maps on $\mathbb{R}^{2 M}$ with values in $\mathbb{R}^{2}, F$ is a real-valued linear map on $\mathbb{R}^{M}$, and $G$ is a real-valued quadratic map on $\mathbb{R}^{2 M}$. With the choice $\eta=\max _{i=1, \ldots, M}\left\|A_{i}\right\|$ we find that

$$
\left\|E_{\nu}\right\| \leqslant \sum_{k=2}^{m-1} \eta^{k-1}+\sum_{k=m+1}^{\infty} 2 \eta^{k-1} \leqslant \frac{\eta}{1-\eta}
$$

and

$$
\|F\| \leqslant \sum_{k=1}^{\infty} 2 \eta^{2 k}=\frac{2 \eta^{2}}{1-\eta^{2}} .
$$

(Observe that it is only necessary to ensure the invertibility of $E$ and $F$ in order to perform the change of variables (6.14) in (6.13). No restriction on $G$ is needed.) The restriction $\eta<\frac{1}{2}$ guarantees (6.15) and (6.16) and completes the proof of the lemma.

To prove Theorems 1.18(ii) and 1.19(ii) we use the following well-known connection between Hausdorff dimension and measures with finite energy.

Proposition 6.19. If $A$ is a subset of a complete metric space $(X, d)$ which supports a Borel measure $\nu$ with $0<\nu(A)<\infty$ whose s-energy

$$
\iint d(x, y)^{-s} d \nu(x) d \nu(y)
$$

is finite, then $\operatorname{dim} A \geqslant s$. 
See, for example, [19, Theorem 8.7] or [9, Corollary 6.6].

The following lemma is the Heisenberg version of Lemma 5.2 in [10]. Its proof, which uses Proposition 6.19, is entirely analogous to the proof of the result from [10] and will be omitted.

Lemma 6.20. Let $\mu$ be a Borel measure on $\Sigma$ with $0<\mu(\Sigma)<\infty$ for which

$$
\int_{\Sigma} \int_{\Sigma} \int_{B_{H}(0, R)^{M}} \frac{d \widetilde{b} d \mu(w) d \mu\left(w^{\prime}\right)}{d_{H}\left(p_{H}(\widetilde{b}, w), p_{H}\left(\widetilde{b}, w^{\prime}\right)\right)^{t}}<\infty
$$

for some $t<4$ and some $R<\infty$. Then

$$
\operatorname{dim}_{H} K_{H}(b, \tau) \geqslant t
$$

for almost every $\widetilde{b}=(b, \tau) \in B_{H}(0, R)^{M} \subset \mathbb{H}^{M}$.

Proof of Theorem 1.19(ii). Fix $R>0$ and choose $t \neq 1,3$ so that $0<t<\min \left\{4, \widetilde{s}_{H}(\mathcal{A})\right\}$. We will verify the assumptions of Lemma 6.20 for such a choice of $t$.

Fix $s$ so that $t<s<\min \left\{4, \widetilde{s}_{H}(\mathcal{A})\right\}$. Then $\widetilde{\mathcal{M}}_{H}^{s}(\Sigma)=\infty$. By Lemma 5.11 , there exist a compact set $C_{0} \subset \Sigma$ and a constant $C<\infty$ so that $0<\widetilde{\mathcal{M}}_{H}^{s}\left(C_{0}\right)<\infty$ and

$$
\mu\left(\Sigma_{w}\right) \leqslant C \psi^{s}\left(A_{w}\right), \quad \text { for } w \in W,
$$

where $\mu$ is the Borel measure on $\Sigma$ given by $\mu(A):=\widetilde{\mathcal{M}}_{H}^{s}\left(C_{0} \cap A\right)$. By Lemma 6.12,

$$
\begin{aligned}
I & :=\int_{\Sigma} \int_{\Sigma} \int_{B_{H}(0, R)^{M}} \frac{d \tilde{b} d \mu(w) d \mu\left(w^{\prime}\right)}{d_{H}\left(p_{H}(\widetilde{b}, w), p_{H}\left(\widetilde{b}, w^{\prime}\right)\right)^{t}} \\
& \leqslant C \int_{\Sigma} \int_{\Sigma} \frac{d \mu(w) d \mu\left(w^{\prime}\right)}{\psi^{t}\left(A_{w \wedge w^{\prime}}\right)}
\end{aligned}
$$

and by the definition of the cylinder sets $\Sigma_{w}$ and (6.21) we obtain

$$
\begin{aligned}
I \leqslant C \sum_{\alpha \in W} \sum_{i \neq j} \frac{\mu\left(\Sigma_{\alpha i}\right) \mu\left(\Sigma_{\alpha j}\right)}{\psi^{t}\left(A_{\alpha}\right)} & \leqslant C \sum_{w \in W} \frac{\mu\left(\Sigma_{w}\right)^{2}}{\psi^{t}\left(A_{w}\right)} \\
& \leqslant C \sum_{m=1}^{\infty} \sum_{w \in W_{m}} \frac{\psi^{s}\left(A_{w}\right) \mu\left(\Sigma_{w}\right)}{\psi^{t}\left(A_{w}\right)} .
\end{aligned}
$$

From the definition (5.7) of the Heisenberg singular value function, we see that

$$
\psi^{s}\left(A_{w}\right) \leqslant \psi^{t}\left(A_{w}\right) \alpha_{1}\left(A_{w}\right)^{s-t}
$$

for all contractive linear $A_{w}$. The submultiplicativity of the singular value function gives

$$
\alpha_{1}\left(A_{w}\right) \leqslant \alpha_{1}\left(A_{w_{1}}\right) \ldots \alpha_{1}\left(A_{w_{m}}\right), \quad \text { where } w=w_{1} \ldots w_{m} .
$$

Fix $a<1$ so that $\alpha_{1}\left(A_{i}\right) \leqslant a$ for all $i$. Then

$$
\psi^{s}\left(A_{w}\right) \leqslant a^{m(s-t)} \psi^{t}\left(A_{w}\right)
$$

and

$$
I \leqslant C \sum_{m=1}^{\infty} a^{m(s-t)} \sum_{w \in W_{m}} \mu\left(\Sigma_{w}\right) \leqslant C \mu(\Sigma)<\infty
$$

since $a<1, t<s$ and $\mu(\Sigma)=\widetilde{\mathcal{M}}_{H}^{s}\left(C_{0}\right)<\infty$. By Lemma 6.20 we conclude that 
$\operatorname{dim}_{H} K_{H}(b, \tau) \geqslant t$ for almost every $\widetilde{b}=(b, \tau) \in B_{H}(0, R)^{M}$. Letting $R \rightarrow \infty$ gives the result for almost every $\widetilde{b} \in \mathbb{H}^{M}$, and letting $t \nearrow \min \left\{4, \widetilde{s}_{H}(\mathcal{A})\right\}$ through values in $(0,4) \backslash\{1,3\}$ finishes the proof.

For the Euclidean case (Theorem 1.18(ii)) the derivation is similar. It uses the following modified versions of Lemmas 6.6 and 6.12 .

Lemma 6.22. Let $0<s<3$ be non-integral. For each $R>0$ there exists a constant $C$ depending only on $R$ and $s$ so that

$$
\int_{B_{E}(0, R)} \frac{d p}{\left|\widetilde{A}_{b}(p)\right|_{E}^{s}} \leqslant \frac{C}{\varphi^{s}\left(\widetilde{A}_{b}\right)} .
$$

Lemma 6.23. Assume that $\left\|A_{i}\right\|<\frac{1}{2}$ for each $i$. For $0<s<3$ non-integral and $R>0$, there exists a constant $C=C(R, s)$ so that

$$
\int_{B_{E}(0, R)^{M}} \frac{d \widetilde{b}}{\left|p_{H}(\widetilde{b}, w)-p_{H}\left(\widetilde{b}, w^{\prime}\right)\right|_{E}^{s}} \leqslant \frac{C}{\varphi^{s}\left(\widetilde{A}_{w \wedge w^{\prime}, b_{w \wedge w^{\prime}}}\right)}
$$

for all $w, w^{\prime} \in \Sigma$.

The proofs are easy variations on the proofs of Lemmas 6.6 and 6.12 .

With these lemmas in hand the remainder of the proof of Theorem 1.18(ii) proceeds by analogy with the proof of Theorem 1.19(ii). We omit the details.

\section{Appendix. Eigenvalues of rank 1 perturbations of block diagonal matrices}

This appendix is devoted to discussion and a sketch of an elementary proof of (5.6) using only techniques from linear algebra. The key result (Theorem A.4) concerns deformation of the singular values of block diagonal matrices under rank 1 perturbations. We begin with a classical theorem of Golub [14].

TheOREM A.1 (Golub). Let $D \in \mathbb{R}^{n \times n}$ be a diagonal matrix, with diagonal entries $\lambda_{1}>\lambda_{2}>\ldots>\lambda_{n}$. Let $w=\left(w_{1}, \ldots, w_{n}\right) \in \mathbb{C}^{n}$ be a complex $n$-tuple with non-zero entries, and let $w \otimes w$ be the associated rank 1 matrix whose $(i, j)$ th entry is $\overline{w_{i}} w_{j}$. Finally, let $\epsilon \neq 0$ be real.

Then the eigenvalues of the Hermitian matrix $P(\epsilon)=D+\epsilon w \otimes w$ are the solutions to the equation

$$
1+\epsilon \sum_{i=1}^{n} \frac{\left|w_{i}\right|^{2}}{\lambda_{i}-\lambda}=0
$$

Corollary A.2. If $\epsilon>0$ then the eigenvalues $\lambda_{1}^{\prime}>\ldots>\lambda_{n}^{\prime}$ of $P(\epsilon)$ interlace with the eigenvalues of $D$ in the following sense:

$$
\lambda_{n}<\lambda_{n}^{\prime}<\ldots<\lambda_{1}<\lambda_{1}^{\prime} .
$$

For extensions and further discussion of Golub's theorem, see Anderson [2]. 
Assume that the conditions in Corollary A.2 hold and assume also that $\lambda_{n}=0$. Then $P=\widetilde{A}^{T} \widetilde{A}$, where

$$
\widetilde{A}=\left(\begin{array}{cccc}
\alpha_{1} & & & \\
& \alpha_{2} & & \\
& & \ddots & \\
\sqrt{\epsilon} w_{1} & \sqrt{\epsilon} w_{2} & \cdots & \sqrt{\epsilon} w_{n}
\end{array}\right)
$$

and $\alpha_{i}$ denotes the positive square root of $\lambda_{i}$. The values $\alpha_{1}, \ldots, \alpha_{n-1}, 0$ are the singular values for $A=\sqrt{D}$. Denote by $\alpha_{i}^{\prime}=\sqrt{\lambda_{i}^{\prime}}$, for $i=1, \ldots, n$, the singular values for $P$. Then (A.3) implies the following inequality between the singular value functions for $A$ and $\widetilde{A}$ :

$$
\varphi^{s}(A) \leqslant \varphi^{s}(\widetilde{A}), \quad \text { for } 0 \leqslant s \leqslant n .
$$

This gives some indication of how Theorem A.1 may be applied in the context of Falconer's theory. For the specific application to (5.6), however, we require a version of Golub's result for block diagonal matrices. As we are interested in applications to the first Heisenberg group, we give the following result only in the case $n=3$.

Theorem A.4. Let $A \in \mathbb{R}^{2 \times 2}$ with distinct singular values $0<\alpha_{2}<\alpha_{1}$, let $b \in \mathbb{R}^{2}$ and let $\epsilon \neq 0$. Set

$$
D=\left(\begin{array}{cc}
A & 0 \\
0 & 0
\end{array}\right) \in \mathbb{R}^{3 \times 3}, \quad w=w(A, b)=\left(\begin{array}{ll}
-2(J b)^{T} A & \operatorname{det} A),
\end{array}\right.
$$

and

$$
\widetilde{A}_{b}(\epsilon)=D+\sqrt{\epsilon} e_{3} \otimes w=\left(\begin{array}{cc}
A & \\
-2 \sqrt{\epsilon}(J b)^{T} A & \sqrt{\epsilon} \operatorname{det} A
\end{array}\right),
$$

where $e_{3}=(0,0,1)$.

Let $\lambda \in \mathbb{C} \backslash\left\{\lambda_{1}, \lambda_{2}\right\}$, where $\lambda_{i}=\alpha_{i}^{2}$, for $i=1,2$. Then $\lambda$ is an eigenvalue for $P(\epsilon)=\widetilde{A}_{b}(\epsilon)^{T} \widetilde{A}_{b}(\epsilon)$ if and only if $\lambda$ is a solution to the equation

$$
F(\lambda):=1+\epsilon\left\{\frac{(\operatorname{det} A)^{2}}{-\lambda}+\frac{C_{1}}{\lambda_{1}-\lambda}+\frac{C_{2}}{\lambda_{2}-\lambda}\right\}=0,
$$

where

$$
C_{1}=\frac{4 \lambda_{1}\left(\left|A^{T} J b\right|^{2}-\lambda_{2}|b|^{2}\right)}{\lambda_{1}-\lambda_{2}}
$$

and

$$
C_{2}=\frac{4 \lambda_{2}\left(\lambda_{1}|b|^{2}-\left|A^{T} J b\right|^{2}\right)}{\lambda_{1}-\lambda_{2}} .
$$

Observe that $C_{1}, C_{2} \geqslant 0$ since $\lambda_{1}$ and $\lambda_{2}$ are the eigenvalues of $A^{T} A$.

The proof of this theorem is by direct computation of the characteristic polynomial of $P(\epsilon)$. We omit the details.

A modified version of Theorem A.4 holds in the orthogonal case $\lambda_{1}=\lambda_{2}$; we omit the details. More general versions are presumably true for block diagonal matrices in higher dimensions, but we do not pursue this here. 
We now sketch the application of Theorem A.4 to the proof of (5.6). Observe that $\widetilde{A}_{b}(1)=\widetilde{A}_{b}$, where $\widetilde{A}_{b}$ is given in (2.2). We assume that $b \neq 0$. As before, denote by $\lambda_{1}^{\prime}>\lambda_{2}^{\prime}>\lambda_{3}^{\prime}$ the eigenvalues of $P(1)=\widetilde{A}_{b}^{T} \widetilde{A}_{b}$.

If $J b$ is not an eigenvector for $A^{T} A$, then $C_{1}$ and $C_{2}$ are both positive. Consequently, we may conclude as in Corollary A.2 that the eigenvalues of $P(1)$ and $D$ interlace:

$$
0<\lambda_{3}^{\prime}<\lambda_{2}<\lambda_{2}^{\prime}<\lambda_{1}<\lambda_{1}^{\prime} .
$$

If $\left|A^{T} J b\right|=\alpha_{2}|b|$ then $C_{1}=0, C_{2}=4 \lambda_{2}|b|^{2}>0$ and

$$
0<\lambda_{3}^{\prime} \leqslant \lambda_{2} \leqslant \lambda_{2}^{\prime}<\lambda_{1}<\lambda_{1}^{\prime} \text {. }
$$

Similarly, if $\left|A^{T} J b\right|=\alpha_{1}|b|$ then $C_{2}=0, C_{1}=4 \lambda_{1}|b|^{2}>0$ and

$$
0<\lambda_{3}^{\prime}<\lambda_{2}<\lambda_{2}^{\prime} \leqslant \lambda_{1} \leqslant \lambda_{1}^{\prime} \text {. }
$$

In all cases, we find that

$$
\lambda_{1} \leqslant \lambda_{1}^{\prime} \quad \text { and } \quad \lambda_{2} \leqslant \lambda_{2}^{\prime}
$$

The function $F(\lambda)$ in (A.5) is equal to

$$
\frac{\operatorname{det}(P(\epsilon)-\lambda I)}{\operatorname{det}(D-\lambda I)}=\frac{\operatorname{det}(P-\lambda I)}{(-\lambda)\left(\lambda_{1}-\lambda\right)\left(\lambda_{2}-\lambda\right)} .
$$

From the expression for $F(\lambda)$ in (A.5), the product of the eigenvalues of $P(1)$ is

$$
\lambda_{1}^{\prime} \lambda_{2}^{\prime} \lambda_{3}^{\prime}=(\operatorname{det} A)^{2}=\lambda_{1}^{2} \lambda_{2}^{2}
$$

Since the singular values of $\widetilde{A}_{0}$ are $\alpha_{1}, \alpha_{2}$ and $\alpha_{1} \alpha_{2}$, it follows from (A.6), (A.7) and the definition of the singular value function in (5.5) that

$$
\varphi^{s}\left(\widetilde{A}_{0}\right) \leqslant \varphi^{s}\left(\widetilde{A}_{b}\right)
$$

for all $s \geqslant 0$. Thus the critical exponents satisfy the inequality

$$
\widetilde{s}_{E}(\mathcal{A}) \leqslant \widetilde{s}_{E}(b ; \mathcal{A})
$$

as desired. This concludes the proof of (5.6).

Acknowledgements. We are grateful to Regula Hofer-Isenegger for the MAPLE code which produced the image of the Heisenberg square in Figure 1.2. We also wish to thank the reviewer for helpful comments and suggestions which improved the presentation.

\section{References}

1. L. Ambrosio and B. Kirchneim, 'Rectifiable sets in metric and Banach spaces', Math. Ann. 318 (2000) $527-555$.

2. J. AndERSON, 'A secular equation for the eigenvalues of a diagonal matrix perturbation', Linear Algebra Appl. 246 (1996) 49-70.

3. Z. M. BALOGH, 'Hausdorff dimension distribution of quasiconformal mappings on the Heisenberg group', J. Anal. Math. 83 (2001) 289-312.

4. Z. M. Balogh, R. Hofer-Isenegger and J. T. Tyson, 'Lifts of Lipschitz maps and horizontal fractals in the Heisenberg group', Ergodic Theory Dynam. Systems, to appear.

5. Z. M. Balogh, M. Rickly and F. Serra-Cassano, 'Comparison of Hausdorff measures with respect to the Euclidean and Heisenberg metric', Publ. Mat. 47 (2003) 237-259. 
6. Z. M. Balogh and J. T. Tyson, 'Polar coordinates on Carnot groups', Math. Z. 241 (2002) 697-730.

7. C. BANDT and S. Graf, 'Self-similar sets. VII. A characterization of self-similar fractals with positive Hausdorff measure', Proc. Amer. Math. Soc. 114 (1992) 995-1001.

8. G. A. EDGAR, 'Fractal dimension of self-affine sets: some examples', Measure theory, Oberwolfach, 1990, Rend. Circ. Mat. Palermo (2) Suppl. 28 (1992) 341-358.

9. K. J. Falconer, The geometry of fractal sets, Cambridge Tracts in Mathematics 85 (Cambridge University Press, 1986).

10. K. J. Falconer, 'The Hausdorff dimension of self-affine fractals', Math. Proc. Cambridge Philos. Soc. 103 (1988) 339-350.

11. K. J. Falconer, 'The dimension of self-affine fractals. II', Math. Proc. Cambridge Philos. Soc. 111 (1992) 169-179.

12. B. Franchi, R. Serapioni and F. Serra-Cassano, 'Rectifiability and perimeter in the Heisenberg group', Math. Ann. 321 (2001) 479-531.

13. N. Garofalo and D.-M. Nhieu, 'Isoperimetric and Sobolev inequalities for CarnotCarathéodory spaces and the existence of minimal surfaces', Comm. Pure Appl. Math. 49 (1996) 1081-1144.

14. G. H. Golub, 'Some modified matrix eigenvalue problems', SIAM Rev. 15 (1973) 318-334.

15. M. Gromov, 'Carnot-Carathéodory spaces seen from within', Sub-Riemannian geometry (ed. A. Bellä̈che and J. J. Risler), Progress in Mathematics 144 (Birkhäuser, Basel, 1996) $79-323$.

16. J. E. Hutchinson, 'Fractals and self-similarity', Indiana Univ. Math. J. 30 (1981) 713-747.

17. J. Kigami, Analysis on fractals, Cambridge Tracts in Mathematics 143 (Cambridge University Press, 2001).

18. B. KirCheim and F. SERRA-CASSANo, 'Rectifiability and parametrization of intrinsic regular surfaces in the Heisenberg group', Ann. Scuola Norm. Sup. Pisa Cl. Sci. (5) 3 (2004) 871-896.

19. P. Mattila, Geometry of sets and measures in Euclidean spaces, Cambridge Studies in Advanced Mathematics 44 (Cambridge University Press, 1995).

20. P. A. P. Moran, 'Additive functions of intervals and Hausdorff measure', Proc. Cambridge Philos. Soc. 42 (1946) 15-23.

21. C. A. Rogers, Hausdorff measures (Cambridge University Press, London, 1970).

22. A. Schief, 'Separation properties for self-similar sets', Proc. Amer. Math. Soc. 122 (1994) $111-115$.

23. B. Solomyak, 'Measure and dimension for some fractal families', Math. Proc. Cambridge Philos. Soc. 124 (1998) 531-546.

24. R. S. Strichartz, 'Self-similarity on nilpotent Lie groups', Geometric analysis, Philadelphia, PA, 1991 (ed. E. L. Grinberg), Contemporary Mathematics 140 (American Mathematical Society, Providence, RI, 1992) 123-157.

25. R. S. Strichartz, 'Self-similarity in harmonic analysis', J. Fourier Anal. Appl. 1 (1994) $1-37$.

Zoltán M. Balogh

Department of Mathematics

University of Bern

Sidlerstrasse 5

3012 Bern

Switzerland

zoltan.balogh@math-stat.unibe.ch
Jeremy T. Tyson

Department of Mathematics

University of Illinois

1409 W. Green Street

Urbana

IL 61801

USA

tyson@math.uiuc.edu 